International Journal of Pure and Applied Mathematics

Volume 95 No. 2 2014, 181-208

ISSN: 1311-8080 (printed version); ISSN: 1314-3395 (on-line version)

url: http://www.ijpam.eu

doi: http://dx.doi.org/10.12732/ijpam.v95i2.6

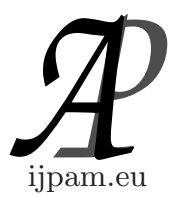

\title{
THE MAPPING METHOD FOR SOLVING A NEW MODEL OF NONLINEAR PARTIAL DIFFERENTIAL EQUATION
}

\author{
A. Maher ${ }^{1}$, H.M. El-Hawary ${ }^{2}$, M.S. Al-Amry ${ }^{3} \S$ \\ ${ }^{1,2}$ Department of Mathematics \\ Faculty of Science \\ Assiut University \\ 71516, EGYPT \\ ${ }^{3}$ Department of Mathematics \\ Faculty of Science and Education \\ Aden University \\ YEMEN
}

\begin{abstract}
In this paper we present a new model of Benjamin-Bona-Mahony (BBM) equation. Then we apply the mapping method to solve the new model. Exact travelling wave solutions are obtained and expressed in terms of hyperbolic functions, trigonometric functions, rational functions and elliptic functions.
\end{abstract}

Key Words: mapping method, exact solutions, combined Benjamin-BonaMahony (BBM), modified Benjamin-Bona-Mahony (mBBM) equation

\section{Introduction}

In recent years, directly searching for exact solutions of nonlinear partial differential equations (PDEs) has become more and more attractive field in different branches of physics and applied mathematics. These equations appear in con-

Received: April 13, 2014

(c) 2014 Academic Publications, Ltd.

$\S$ Correspondence author url: www.acadpubl.eu 
densed matter, solid state physics, fluid mechanics, chemical kinetics, plasma physics, nonlinear optics, propagation of fluxions in Josephson junctions, theory of turbulence, ocean dynamics, biophysics and star formation and many others.

In order to get exact solutions directly, many powerful methods have been introduced such as the $\left(\frac{G^{\prime}}{G}\right)$-expansion method [1], inverse scattering method $[2,3]$, Hirota's bilinear method [4,5], the tanh method [6,7], the sine-cosine method [8,9], Backlund transformation method [10,11], the homogeneous balance [12,13], Darboux transformation [14], the Jacobi elliptic function expansion method [15].

Recently, Yan-Ze Peng [16] introduced a new approach, namely, the mapping method for a reliable treatment of the nonlinear wave equations. The useful mapping method is then widely used by many authors $[17,18,19]$.

Benjamin-Bona-Mahony (BBM) equation [20], is nonlinear wave equations modeling unidirectional propagation of long wave in dispersive media. It is originally derived by using a Pade' $(2,2)$ approximation of the phase velocity that arises in linear wave theory [21].

\section{Mapping Method}

Consider the general nonlinear partial differential equations (PDEs), say, in two variables,

$$
P\left(u, u_{x}, u_{t}, u_{x x}, u_{x t}, \ldots\right)=0 .
$$

Let $u(x, t)=u(\xi), \xi=\mu(x-c t)$ then equation (1) reduces to a nonlinear ordinary differential equation (ODE)

$$
Q\left(u, u^{\prime}, u^{\prime \prime}, \ldots\right)=0
$$

Assume the solution of equation (2) takes the form

$$
u(x, t)=u(\xi)=a_{0}+\sum_{i=1}^{m} a_{i}(f(\xi))^{i}+b_{i}(f(\xi))^{-i},
$$

where the coefficients $a_{i}(i=0,1,2, \ldots, m), \mu, c$ are constants to be determined, and $f=f(\xi)$ satisfies a nonlinear ordinary differential equation

$$
\frac{d f(\xi)}{d \xi}=\sqrt{p f^{2}(\xi)+\frac{1}{2} q f^{4}(\xi)+r}, p, q, r \in R
$$


where the coefficients $a_{0}, a_{i}, b_{i}(i=1,2, \ldots m), \mu$ and $c$ are constants to be determined and $f=f(\xi)$ satisfies (4), the parameter $m$ will be found by balancing the highest-order nonlinear terms with the highest-order partial derivative term in the given equation. Substituting (3) into (2), using (4) repeatedly, and setting the coefficients of the each order of $f^{i}(\xi), f^{i}(\xi) \sqrt{p f^{2}(\xi)+\frac{1}{2} q f^{4}(\xi)+r}$ to zero, we obtain a set of nonlinear algebraic equations for $a_{0}, a_{i}, b_{i}(i=1,2, \ldots n), \mu, c$. With the aid of the computer program Maple, we can solve the set of nonlinear algebraic equations and obtain all the constants $a_{0}, a_{i}, b_{i}(i=1,2, \ldots n), \mu$ and c. The ODE (4) has the following solutions

1. $f(\xi)=\operatorname{sech}(\xi),[p=1, q=-2, r=0]$,

2. $f(\xi)=\tanh (\xi),[p=-2, q=2, r=1]$,

3. $f(\xi)=\frac{1}{2} \tanh (2 \xi)$ or $\frac{1}{2} \operatorname{coth}(2 \xi),[p=-8, q=32, r=1]$,

4. $f(\xi)=\frac{1}{2} \tan (2 \xi)$, or $-\frac{1}{2} \cot (2 \xi),[p=8, q=32, r=1]$,

5. $f(\xi)=\operatorname{sn} \xi,\left[p=-\left(k^{2}+1\right), q=2 k^{2}, r=1\right]$,

6. $f(\xi)=\mathrm{ns} \xi,\left[p=-\left(k^{2}+1\right), q=2, r=k^{2}\right]$,

7. $f(\xi)=\operatorname{cd} \xi,\left[p=-\left(k^{2}+1\right), q=2 k^{2}, r=1\right]$,

8. $f(\xi)=\operatorname{dc} \xi,\left[p=-\left(k^{2}+1\right), q=2, r=k^{2}\right]$,

9. $f(\xi)=\operatorname{cn} \xi,\left[p=2 k^{2}-1, q=-2 k^{2}, r=1-k^{2}\right]$,

10. $f(\xi)=\mathrm{nc} \xi,\left[p=2 k^{2}-1, q=2\left(1-k^{2}\right), r=-k^{2}\right]$,

11. $f(\xi)=\operatorname{dn} \xi,\left[p=2-k^{2}, q=-2, r=-\left(1-k^{2}\right)\right]$,

12. $f(\xi)=\mathrm{nd} \xi,\left[p=2-k^{2}, q=2\left(k^{2}-1\right), r=-1\right]$,

13. $f(\xi)=\operatorname{cs} \xi,\left[p=2-k^{2}, q=2, r=1-k^{2}\right]$,

14. $f(\xi)=\operatorname{sc} \xi,\left[p=2-k^{2}, q=2\left(1-k^{2}\right), r=1\right]$,

15. $f(\xi)=\mathrm{ds} \xi,\left[p=-1+2 k^{2}, q=2, r=-k^{2}\left(1-k^{2}\right)\right]$,

16. $f(\xi)=\operatorname{sd} \xi,\left[p=-1+2 k^{2}, q=2 k^{2}\left(k^{2}-1\right), r=1\right]$,

17. $f(\xi)=\operatorname{sc} \xi \pm \operatorname{nc} \xi,\left[p=\frac{1+k^{2}}{2}, q=\frac{1-k^{2}}{2}, r=\frac{1-k^{2}}{4}\right]$, 
18. $f(\xi)=\frac{\operatorname{sn} \xi}{1 \pm \operatorname{dn} \xi},\left[p=\frac{k^{2}-2}{2}, q=\frac{k^{2}}{2}, r=\frac{1}{4}\right]$,

19. $f(\xi)=\frac{\operatorname{dn} \xi}{1 \pm k \operatorname{sn} \xi},\left[p=\frac{k^{2}+1}{2}, q=\frac{k^{2}-1}{2}, r=\frac{1-k^{2}}{4}\right]$,

20. $f(\xi)=k \operatorname{cn} \xi \pm \operatorname{dn} \xi,\left[p=\frac{k^{2}+1}{2}, q=\frac{-1}{2}, r=\frac{-\left(1-k^{2}\right)^{2}}{4}\right]$,

21. $f(\xi)=\frac{\mathrm{cn} \xi}{1 \pm \operatorname{sn} \xi},\left[p=\frac{k^{2}+1}{2}, q=\frac{1-k^{2}}{2}, r=\frac{1-k^{2}}{4}\right]$,

22. $f(\xi)=k \operatorname{sn} \xi \pm i \operatorname{dn} \xi,\left[p=\frac{1-2 k^{2}}{2}, q=\frac{1}{2}, r=\frac{k^{2}}{4}\right]$,

23. $f(\xi)=k \operatorname{sn} \xi \pm i \operatorname{cn} \xi,\left[p=\frac{k^{2}-2}{2}, q=\frac{k^{2}}{2}, r=\frac{k^{2}}{4}\right]$,

24. $f(\xi)=\mathrm{ns} \xi \pm \mathrm{ds} \xi, \quad\left[p=\frac{k^{2}-2}{2}, q=\frac{1}{2}, r=\frac{k^{4}}{4}\right]$,

25. $f(\xi)=\mathrm{ns} \xi-\operatorname{cs} \xi,\left[p=\frac{1-2 k^{2}}{2}, q=\frac{1}{2}, r=\frac{1}{4}\right]$,

26. $f(\xi)=\frac{\mathrm{cn} \xi}{\sqrt{1-k^{2}} \operatorname{sn} \xi \pm \operatorname{dn} \xi},\left[p=\frac{1-2 k^{2}}{2}, q=\frac{1}{2}, r=\frac{1}{4}\right]$,

27. $f(\xi)=\frac{\operatorname{sn} \xi}{\operatorname{cn} \xi \pm \operatorname{dn} \xi},\left[p=\frac{1+k^{2}}{2}, q=\frac{\left(1-k^{2}\right)^{2}}{2}, r=\frac{1}{4}\right]$,

28. $f(\xi)=\frac{\mathrm{cn} \xi}{\sqrt{1-k^{2}} \pm \operatorname{dn} \xi},\left[p=\frac{k^{2}-2}{2}, q=\frac{k^{2}}{2}, r=\frac{1}{4}\right]$,

29. $f(\xi)=\frac{-1}{\sqrt{\frac{c}{2}} \xi},[p=0, q=c, r=0]$,

30. $f(\xi)=e^{\xi}, \quad[p=1, q=0, r=0]$.

The multiple exact special solutions of nonlinear partial differential equation (1) are obtained by making use of (3) and the solutions of ODE(4).

\section{Application}

In this section, we present our proposed equation, namely, a combined BenjaminBona-Mahony (BBM) and modified Benjamin-Bona-Mahony (mBBM) equation as the form

$$
u_{t}(x, t)+u_{x}(x, t)+\beta u(x, t) u_{x}(x, t)+\beta u^{2}(x, t) u_{x}(x, t)-\alpha u_{x x t}(x, t)=0
$$


Where

$$
u_{t}(x, t)+u_{x}(x, t)+\beta u(x, t) u_{x}(x, t)-\alpha u_{x x t}(x, t)=0, \quad \beta>0, \alpha \neq 0
$$

is Benjamin-Bona-Mahony (BBM) equation

and

$$
u_{t}(x, t)+u_{x}(x, t)+\beta u^{2}(x, t) u_{x}(x, t)-\alpha u_{x x t}(x, t)=0
$$

is modified Benjamin-Bona-Mahony (mBBM) equation.

Substituting $u(x, t)=u(\xi), \xi=\lambda(x-c t)$ in (5) and integrating once yields

$$
(1-c) u(\xi)+\frac{\beta}{2}(u(\xi))^{2}+\frac{\beta}{3}(u(\xi))^{3}+c \lambda^{2} \alpha \frac{d^{2}}{d \xi^{2}} u(\xi)=0 .
$$

Balancing the order of the nonlinear term $u^{3}$ with the highest derivative $u^{\prime \prime}$ gives $3 m=m+2$ that gives $m=1$. Now, we apply the mapping method to solve our equation. consequently we get the original solutions for our new equation, as the following:

Assume, the solution of (8) has the form

$$
u(\xi)=a_{0}+a_{1} f(\xi)+b_{1} f(\xi)^{-1}
$$

Where

$$
\frac{d f(\xi)}{d \xi}=\sqrt{p f^{2}(\xi)+\frac{1}{2} q f^{4}(\xi)+r}, p, q, r \in R .
$$

Substituting (9) in (8) and using (10), collecting the coefficients of each power of $f^{i}, 0 \leq i \leq 6$, setting each coefficient to zero, and solving the resulting system, obtain the following sets of solutions.

1.

$$
a_{0}=0, a_{1}=b_{1}=0, c=c, \lambda=\lambda,
$$

2 .

$$
a_{0}=a_{0}, a_{1}=b_{1}=0, c=c, \lambda=\lambda
$$

3.

$$
a_{0}=\frac{-1}{2}, a_{1}=\sqrt{-\frac{q}{4 p}}, b_{1}=0, c=1-\frac{\beta}{6}, \lambda= \pm \sqrt{\frac{\beta}{-12 \alpha p+2 \alpha \beta p}}
$$


4.

$$
a_{0}=\frac{-1}{2}, a_{1}=-\sqrt{-\frac{q}{4 p}}, b_{1}=0, c=1-\frac{\beta}{6}, \lambda= \pm \sqrt{\frac{\beta}{-12 \alpha p+2 \alpha \beta p}},
$$

5.

$$
a_{0}=\frac{-1}{2}, a_{1}=0, b_{1}=\sqrt{-\frac{r}{2 p}}, c=1-\frac{\beta}{6}, \lambda= \pm \sqrt{\frac{\beta}{-12 \alpha p+2 \alpha \beta p}},
$$

6.

$$
a_{0}=\frac{-1}{2}, a_{1}=0, b_{1}=-\sqrt{-\frac{r}{2 p}}, c=1-\frac{\beta}{6}, \lambda= \pm \sqrt{\frac{\beta}{-12 \alpha p+2 \alpha \beta p}}
$$

7.

$$
\begin{gathered}
a_{0}=\frac{-1}{2}, a_{1}=\frac{1}{2} \sqrt{\frac{q(-p+3 \sqrt{2 r q})}{p^{2}-18 r q}}, b_{1}=\frac{1}{2} \frac{-6 r q+p \sqrt{2 r q}}{\sqrt{\frac{q(-p+3 \sqrt{2 r q})}{p^{2}-18 r q}}\left(p^{2}-18 r q\right)}, \\
c=1-\frac{\beta}{6}, \lambda= \pm \frac{\sqrt{\alpha \beta\left(p^{2} \beta-6 p^{2}+108 r q-18 r q \beta\right)(-p+3 \sqrt{2 r q})}}{\alpha\left(p^{2} \beta-6 p^{2}+108 r q-18 r q \beta\right)}
\end{gathered}
$$

8.

$$
\begin{gathered}
a_{0}=\frac{-1}{2}, a_{1}=-\frac{1}{2} \sqrt{\frac{q(-p+3 \sqrt{2 r q})}{p^{2}-18 r q}}, b_{1}=\frac{1}{2} \frac{6 r q-p \sqrt{2 r q}}{\sqrt{\frac{q(-p+3 \sqrt{2 r q})}{p^{2}-18 r q}}\left(p^{2}-18 r q\right)}, \\
c=1-\frac{\beta}{6}, \lambda= \pm \frac{\sqrt{\alpha \beta\left(p^{2} \beta-6 p^{2}+108 r q-18 r q \beta\right)(-p+3 \sqrt{2 r q})}}{\alpha\left(p^{2} \beta-6 p^{2}+108 r q-18 r q \beta\right)}
\end{gathered}
$$

9.

$$
a_{0}=\frac{-1}{2}, a_{1}=\frac{1}{2} \sqrt{\frac{q(p+3 \sqrt{2 r q})}{-p^{2}+18 r q}}, b_{1}=-\frac{1}{2} \frac{6 r q+p \sqrt{2 r q}}{\sqrt{\frac{q(p+3 \sqrt{2 r q})}{18 r q-p^{2}}}\left(p^{2}-18 r q\right)}
$$




$$
c=1-\frac{\beta}{6}, \lambda= \pm \frac{\sqrt{-\alpha \beta\left(p^{2} \beta-6 p^{2}+108 r q-18 r q \beta\right)(p+3 \sqrt{2 r q})}}{\alpha\left(p^{2} \beta-6 p^{2}+108 r q-18 r q \beta\right)},
$$

10.

$$
\begin{gathered}
a_{0}=\frac{-1}{2}, a_{1}=-\frac{1}{2} \sqrt{\frac{q(p+3 \sqrt{2 r q})}{-p^{2}+18 r q}}, b_{1}=\frac{1}{2} \frac{6 r q+p \sqrt{2 r q}}{\sqrt{\frac{q(p+3 \sqrt{2 r q})}{18 r q-p^{2}}}\left(p^{2}-18 r q\right)}, \\
c=1-\frac{\beta}{6}, \lambda= \pm \frac{\sqrt{-\alpha \beta\left(p^{2} \beta-6 p^{2}+108 r q-18 r q \beta\right)(p+3 \sqrt{2 r q})}}{\alpha\left(p^{2} \beta-6 p^{2}+108 r q-18 r q \beta\right)} .
\end{gathered}
$$

Using (9), the solution of (10) when $p=1, q=-2, r=0$, and the sets of solutions (1)-(10), we get

$$
\begin{aligned}
& u_{1}(x, t)=0, \\
& u_{2}(x, t)=a_{0} \quad \forall a_{0} \in R,
\end{aligned}
$$

for $\frac{-\beta}{\alpha(\beta-6)}>0$, we obtain

$u_{3,4}(x, t)=-\frac{1}{2} \pm \frac{1}{\sqrt{2}} \operatorname{sech}\left(\sqrt{2} \sqrt{\frac{-\beta}{\alpha(\beta-6)}}\left(x-\left(1-\frac{\beta}{6}\right) t\right)\right)$,

for $\frac{-\beta}{\alpha(\beta-6)}<0$, we get

$u_{5,6}(x, t)=-\frac{1}{2} \pm \frac{1}{\sqrt{2}} \sec \left(\sqrt{2} \sqrt{\frac{\beta}{\alpha(\beta-6)}}\left(x-\left(1-\frac{\beta}{6}\right) t\right)\right)$.

Using (9), the solution of (10) when $p=-2, q=2, r=1$, and the sets of solutions (3)-(10), we get

$$
\begin{aligned}
& \text { for } \frac{\beta}{\alpha(\beta-6)}>0, \text { we obtain } \\
& u_{7,8}(x, t)=-\frac{1}{2} \pm \frac{1}{2} \tanh \left(\frac{1}{2} \sqrt{\frac{\beta}{\alpha(\beta-6)}}\left(x-\left(1-\frac{\beta}{6}\right) t\right)\right), \\
& u_{9,10}(x, t)=-\frac{1}{2} \pm \frac{1}{2} \operatorname{coth}\left(\frac{1}{2} \sqrt{\frac{\beta}{\alpha(\beta-6)}}\left(x-\left(1-\frac{\beta}{6}\right) t\right)\right), \\
& u_{11,12}(x, t)=-\frac{1}{2} \pm \frac{1}{2 \sqrt{2}} \tan \left(\frac{1}{2 \sqrt{2}} \sqrt{\frac{\beta}{\alpha(\beta-6)}}\left(x-\left(1-\frac{\beta}{6}\right) t\right)\right) \\
& \mp \frac{1}{2 \sqrt{2}} \cot \left(\frac{1}{2 \sqrt{2}} \sqrt{\frac{\beta}{\alpha(\beta-6)}}\left(x-\left(1-\frac{\beta}{6}\right) t\right)\right), \\
& u_{13,14}(x, t)=-\frac{1}{2} \pm \frac{1}{4} \tanh \left(\frac{1}{4} \sqrt{\frac{\beta}{\alpha(\beta-6)}}\left(x-\left(1-\frac{\beta}{6}\right) t\right)\right) \\
& \pm \frac{1}{4} \operatorname{coth}\left(\frac{1}{4} \sqrt{\frac{\beta}{\alpha(\beta-6)}}\left(x-\left(1-\frac{\beta}{6}\right) t\right)\right) .
\end{aligned}
$$




$$
\begin{aligned}
& \text { for } \frac{\beta}{\alpha(\beta-6)}<0 \text {, we get } \\
& u_{15,16}(x, t)=-\frac{1}{2} \pm \frac{i}{2} \tan \left(\frac{1}{2} \sqrt{\frac{-\beta}{\alpha(\beta-6)}}\left(x-\left(1-\frac{\beta}{6}\right) t\right)\right), \\
& u_{17,18}(x, t)=-\frac{1}{2} \pm \frac{i}{2} \cot \left(\frac{1}{2} \sqrt{\frac{-\beta}{\alpha(\beta-6)}}\left(x-\left(1-\frac{\beta}{6}\right) t\right)\right), \\
& u_{19,20}(x, t)=-\frac{1}{2} \pm \frac{i}{2 \sqrt{2}} \tanh \left(\frac{1}{2 \sqrt{2}} \sqrt{\frac{-\beta}{\alpha(\beta-6)}}\left(x-\left(1-\frac{\beta}{6}\right) t\right)\right) \\
& \mp \frac{i}{2 \sqrt{2}} \operatorname{coth}\left(\frac{1}{2 \sqrt{2}} \sqrt{\frac{-\beta}{\alpha(\beta-6)}}\left(x-\left(1-\frac{\beta}{6}\right) t\right)\right), \\
& u_{21,22}(x, t)=-\frac{1}{2} \pm \frac{i}{4} \tan \left(\frac{1}{4} \sqrt{\frac{-\beta}{\alpha(\beta-6)}}\left(x-\left(1-\frac{\beta}{6}\right) t\right)\right) \\
& \pm \frac{i}{4} \cot \left(\frac{1}{4} \sqrt{\frac{-\beta}{\alpha(\beta-6)}}\left(x-\left(1-\frac{\beta}{6}\right) t\right)\right) .
\end{aligned}
$$

Using (9), the solution of (10) when $p=8, q=32, r=1$, and the sets of solutions $(3)-(10)$, we get, $\left[u_{7,8}(x, t), u_{9,10}(x, t), \ldots, u_{21,22}(x, t)\right]$

Using (9), the solution of (10) when $p=-8, q=32, r=1$, and the sets of solutions $(3)-(10)$, we get, $\left[u_{7,8}(x, t), u_{9,10}(x, t), \ldots, u_{21,22}(x, t)\right]$.

Using (9), the solution of (10) when $p=-\left(k^{2}+1\right), q=2 k^{2}, r=1$, and the sets of solutions (3)-(10), we get

$u_{23,24, \ldots, 30}(x, t)=a_{0}+a_{1} \operatorname{sn} \xi+b_{1} \mathrm{~ns} \xi$, where $a_{0}, a_{1}$ and $b_{1}$ are defined in the sets of solutions (3)-(10).

Note that, when $k \rightarrow 1$ we obtain, $\left[u_{7,8}(x, t), u_{9,10}(x, t), \ldots, u_{21,22}(x, t)\right]$, when $k \rightarrow 0$ we obtain for $\frac{\beta}{\alpha(\beta-6)}>0$,

$$
\begin{aligned}
& u_{31,32}(x, t)=-\frac{1}{2} \pm \frac{1}{\sqrt{2}} \csc \left(\frac{1}{\sqrt{2}} \sqrt{\frac{\beta}{\alpha(\beta-6)}}\left(x-\left(1-\frac{\beta}{6}\right) t\right)\right), \\
& \text { for } \frac{\beta}{\alpha(\beta-6)}<0, \\
& u_{33,34}(x, t)=-\frac{1}{2} \pm \frac{i}{\sqrt{2}} \operatorname{csch}\left(\frac{1}{\sqrt{2}} \sqrt{\frac{-\beta}{\alpha(\beta-6)}}\left(x-\left(1-\frac{\beta}{6}\right) t\right)\right) .
\end{aligned}
$$

Using (9), the solution of (10) when $p=-\left(k^{2}+1\right), q=2 k^{2}, r=1$, and the sets of solutions (3)-(10), we get

$u_{35,36, \ldots, 42}(x, t)=a_{0}+a_{1} \mathrm{~cd} \xi+b_{1} \mathrm{dc} \xi$, where $a_{0}, a_{1}$ and $b_{1}$ are defined in the sets of solutions (3)-(10).

Note that, when $k \rightarrow 1$ we obtain constant solutions, when $k \rightarrow 0$ we obtain, $\left[u_{3,4}(x, t)\right.$ and $\left.u_{5,6}(x, t)\right]$.

Using (9), the solution of (10) when $p=-\left(k^{2}+1\right), q=2, r=k^{2}, \quad$ and the sets of solutions (3)-(10), we get 
$u_{43,44, \ldots, 50}(x, t)=a_{0}+a_{1} \mathrm{~ns} \xi+b_{1} \operatorname{sn} \xi$, where $a_{0}, a_{1}$ and $b_{1}$ are defined in the sets of solutions (3)-(10).

Note that, when $k \rightarrow 1$ we obtain, $\left[u_{7,8}(x, t), u_{9,10}(x, t), \ldots, u_{21,22}(x, t)\right]$, when $k \rightarrow 0$ we obtain, $\left[u_{31,32}(x, t)\right.$ and $\left.u_{33,34}(x, t)\right]$.

Using (9), the solution of (10) when $p=-\left(k^{2}+1\right), q=2, r=k^{2}$, and the sets of solutions (3)-(10), we get

$u_{51,52, \ldots, 57}(x, t)=a_{0}+a_{1} \operatorname{dc} \xi+b_{1} \operatorname{cd} \xi$, where $a_{0}, a_{1}$ and $b_{1}$ are defined in the sets of solutions $3-10$.

Note that, when $k \rightarrow 1$ we obtain constant solution, when $k \rightarrow 0$ we obtain $\left[u_{3,4}(x, t)\right.$ and $\left.u_{5,6}(x, t)\right]$.

Using (9), the solution of (10) when $p=2 k^{2}-1, q=-2 k^{2}, r=1-k^{2}$, and the sets of solutions (3)-(10), we get

$u_{58,59, \ldots, 65}(x, t)=a_{0}+a_{1} \operatorname{cn} \xi+b_{1}$ nc $\xi$, where $a_{0}, a_{1}$ and $b_{1}$ are defined in the sets of solutions (3)-(10).

Note that, when $k \rightarrow 1$ we obtain, $\left[u_{3,4}(x, t)\right.$ and $\left.u_{5,6}(x, t)\right]$, when $k \rightarrow 0$ we obtain, $\left[u_{3,4}(x, t)\right.$ and $\left.u_{5,6}(x, t)\right]$.

Using (9), the solution of (10) when $p=2 k^{2}-1, q=2\left(1-k^{2}\right), r=-k^{2}$, and the sets of solutions (3)-(10), we get

$u_{66,67, \ldots, 73}(x, t)=a_{0}+a_{1} \mathrm{nc} \xi+b_{1} \operatorname{cn} \xi$, where $a_{0}, a_{1}$ and $b_{1}$ are defined in the sets of solutions (3)-(10).

Note that, when $k \rightarrow 1$ we obtain, $\left[u_{3,4}(x, t)\right.$ and $\left.u_{5,6}(x, t)\right]$, when $k \rightarrow 0$ we obtain, $\left[u_{3,4}(x, t)\right.$ and $\left.u_{5,6}(x, t)\right]$.

Using (9), the solution of (10) when $p=2-k^{2}, q=-2, r=-\left(1-k^{2}\right)$, and the sets of solutions (3)-(10), we get

$u_{74,75, \ldots, 81}(x, t)=a_{0}+a_{1} \operatorname{dn} \xi+b_{1}$ nd $\xi$, where $a_{0}, a_{1}$ and $b_{1}$ are defined in the sets of solutions (3)-(10).

Note that, when $k \rightarrow 1$ we obtain, $\left[u_{3,4}(x, t)\right.$ and $\left.u_{5,6}(x, t)\right]$, when $k \rightarrow 0$ we obtain constant solutions.

Using (9), the solution of (10) when $p=2-k^{2}, q=2\left(k^{2}-1\right), r=-1$, and the sets of solutions (3)-(10), we get

$u_{82,83, \ldots, 89}(x, t)=a_{0}+a_{1} \operatorname{nd} \xi+b_{1} \operatorname{dn} \xi$, where $a_{0}, a_{1}$ and $b_{1}$ are defined in the sets of solutions (3)-(10).

Note that, when $k \rightarrow 1$ we obtain, $\left[u_{3,4}(x, t)\right.$ and $\left.u_{5,6}(x, t)\right]$, when $k \rightarrow 0$ we obtain constant solutions .

Using (9), the solution of (10) when $p=2-k^{2}, q=2, r=1-k^{2}$, and the sets of solutions (3)-(10), we get 
$u_{90,91, \ldots, 97}(x, t)=a_{0}+a_{1} \operatorname{cs} \xi+b_{1} \operatorname{sc} \xi$, where $a_{0}, a_{1}$ and $b_{1}$ are defined in the sets of solutions (3)-(10).

Note that, when $k \rightarrow 1$ we obtain, $\left[u_{31,32}(x, t)\right.$ and $\left.u_{33,34}(x, t)\right]$, when $k \rightarrow$ 0 we obtain, $\left[u_{7,8}(x, t), u_{9,10}(x, t), \ldots, u_{21,22}(x, t)\right]$.

Using (9), the solution of (10) when $p=2-k^{2}, q=2\left(1-k^{2}\right), r=1$, and the sets of solutions (3)-(10), we get

$u_{98,99, \ldots, 105}(x, t)=a_{0}+a_{1} \operatorname{sc} \xi+b_{1} \operatorname{cs} \xi$, where $a_{0}, a_{1}$ and $b_{1}$ are defined in the sets of solutions (3)-(10).

Note that, when $k \rightarrow 1$ we obtain, $\left[u_{31,32}(x, t)\right.$ and $\left.u_{33,34}(x, t)\right]$, when $k \rightarrow$ 0 we obtain, $\left[u_{7,8}(x, t), u_{9,10}(x, t), \ldots, u_{21,22}(x, t)\right]$.

Using (9), the solution of (10) when $p=-1+2 k^{2}, q=2, r=-k^{2}\left(1-k^{2}\right)$, and the sets of solutions (3)-(10), we get

$u_{106,107, \ldots, 113}(x, t)=a_{0}+a_{1} \mathrm{ds} \xi+b_{1} \mathrm{sd} \xi$, where $a_{0}, a_{1}$ and $b_{1}$ are defined in the sets of solutions (3)-(10).

Note that, when $k \rightarrow 1$ we obtain, $\left[u_{31,32}(x, t)\right.$ and $\left.u_{33,34}(x, t)\right]$, when $k \rightarrow$ 0 we obtain also, $\left[u_{31,32}(x, t)\right.$ and $\left.u_{33,34}(x, t)\right]$.

Using (9), the solution of (10) when $p=-1+2 k^{2}, q=2 k^{2}\left(k^{2}-1\right), r=1$, and the sets of solutions (3)-(10), we get

$u_{114,115, \ldots, 121}(x, t)=a_{0}+a_{1} \mathrm{sd} \xi+b_{1} \mathrm{ds} \xi$, where $a_{0}, a_{1}$ and $b_{1}$ are defined in the sets of solutions (3)-(10).

Note that, when $k \rightarrow 1$ we obtain, $\left[u_{31,32}(x, t)\right.$ and $\left.u_{33,34}(x, t)\right]$, when $k \rightarrow 0$ we obtain also, $\left[u_{31,32}(x, t)\right.$ and $\left.u_{33,34}(x, t)\right]$.

Using (9), the solution of (10) when $p=\frac{1+k^{2}}{2}, q=\frac{1-k^{2}}{2}, r=\frac{1-k^{2}}{4}, \quad$ and the sets of solutions (3)-(10), we get

$u_{122,123, \ldots, 129}(x, t)=a_{0}+a_{1}(\operatorname{sc} \xi \pm \mathrm{nc} \xi)+b_{1} \frac{1}{\operatorname{sc} \xi \pm \mathrm{nc} \xi}$, where $a_{0}, a_{1}$ and $b_{1}$ are defined in the sets of solutions (3)-(10).

Note that, when $k \rightarrow 1$ we obtain constant solutions, when $k \rightarrow 0$ we obtain, for $\frac{\beta}{\alpha(\beta-6)}<0$,

$u_{130,131}(x, t)=-\frac{1}{2}$

$+\frac{i}{2}\left(\tan \left(\sqrt{\frac{-\beta}{\alpha(\beta-6)}}\left(x-\left(1-\frac{\beta}{6}\right) t\right)\right) \pm \sec \left(\sqrt{\frac{-\beta}{\alpha(\beta-6)}}\left(x-\left(1-\frac{\beta}{6}\right) t\right)\right)\right)$,

$u_{132,133}(x, t)=-\frac{1}{2}$

$-\frac{i}{2}\left(\tan \left(\sqrt{\frac{-\beta}{\alpha(\beta-6)}}\left(x-\left(1-\frac{\beta}{6}\right) t\right)\right) \pm \sec \left(\sqrt{\frac{-\beta}{\alpha(\beta-6)}}\left(x-\left(1-\frac{\beta}{6}\right) t\right)\right)\right)$, $u_{134,135}(x, t)=-\frac{1}{2}+$

$\frac{i}{2\left(\tan \left(\sqrt{\frac{-\beta}{\alpha(\beta-6)}}\left(x-\left(1-\frac{\beta}{6}\right) t\right)\right) \pm \sec \left(\sqrt{\frac{-\beta}{\alpha(\beta-6)}}\left(x-\left(1-\frac{\beta}{6}\right) t\right)\right)\right)}$ 
$u_{136,137}(x, t)=-\frac{1}{2}-$

$$
\begin{aligned}
& \frac{i}{2\left(\tan \left(\sqrt{\frac{-\beta}{\alpha(\beta-6)}}\left(x-\left(1-\frac{\beta}{6}\right) t\right)\right) \pm \sec \left(\sqrt{\frac{-\beta}{\alpha(\beta-6)}}\left(x-\left(1-\frac{\beta}{6}\right) t\right)\right)\right)}, \\
& u_{138,139}(x, t)=-\frac{1}{2}+ \\
& \frac{i}{4}\left(\tan \left(\frac{1}{2} \sqrt{\frac{-\beta}{\alpha(\beta-6)}}\left(x-\left(1-\frac{\beta}{6}\right) t\right)\right) \pm \sec \left(\frac{1}{2} \sqrt{\frac{-\beta}{\alpha(\beta-6)}}\left(x-\left(1-\frac{\beta}{6}\right) t\right)\right)\right) \\
& -\frac{\frac{i}{4}}{\tan \left(\frac{1}{2} \sqrt{\frac{-\beta}{\alpha(\beta-6)}}\left(x-\left(1-\frac{\beta}{6}\right) t\right)\right) \pm \sec \left(\frac{1}{2} \sqrt{\frac{-\beta}{\alpha(\beta-6)}}\left(x-\left(1-\frac{\beta}{6}\right) t\right)\right)},
\end{aligned}
$$

$u_{140,141}(x, t)=-\frac{1}{2}-$

$$
\begin{aligned}
& \frac{i}{4}\left(\tan \left(\frac{1}{2} \sqrt{\frac{-\beta}{\alpha(\beta-6)}}\left(x-\left(1-\frac{\beta}{6}\right) t\right)\right) \pm \sec \left(\frac{1}{2} \sqrt{\frac{-\beta}{\alpha(\beta-6)}}\left(x-\left(1-\frac{\beta}{6}\right) t\right)\right)\right) \\
& +\frac{\frac{i}{4}}{\tan \left(\frac{1}{2} \sqrt{\frac{-\beta}{\alpha(\beta-6)}}\left(x-\left(1-\frac{\beta}{6}\right) t\right)\right) \pm \sec \left(\frac{1}{2} \sqrt{\frac{-\beta}{\alpha(\beta-6)}}\left(x-\left(1-\frac{\beta}{6}\right) t\right)\right)},
\end{aligned}
$$

$u_{142,143}(x, t)=-\frac{1}{2}+$

$$
\frac{\sqrt{2}}{4}\left(i \tanh \left(\frac{\sqrt{2}}{2} \sqrt{\frac{-\beta}{\alpha(\beta-6)}}\left(x-\left(1-\frac{\beta}{6}\right) t\right)\right) \pm \operatorname{sech}\left(\frac{\sqrt{2}}{2} \sqrt{\frac{-\beta}{\alpha(\beta-6)}}\left(x-\left(1-\frac{\beta}{6}\right) t\right)\right)\right)
$$$$
+\frac{\frac{\sqrt{2}}{4}}{i \tanh \left(\frac{\sqrt{2}}{2} \sqrt{\frac{-\beta}{\alpha(\beta-6)}}\left(x-\left(1-\frac{\beta}{6}\right) t\right)\right) \pm \operatorname{sech}\left(\frac{\sqrt{2}}{2} \sqrt{\frac{-\beta}{\alpha(\beta-6)}}\left(x-\left(1-\frac{\beta}{6}\right) t\right)\right)},
$$$$
u_{144,145}(x, t)=-\frac{1}{2}-
$$$$
\frac{\sqrt{2}}{4}\left(i \tanh \left(\frac{\sqrt{2}}{2} \sqrt{\frac{-\beta}{\alpha(\beta-6)}}\left(x-\left(1-\frac{\beta}{6}\right) t\right)\right) \pm \operatorname{sech}\left(\frac{\sqrt{2}}{2} \sqrt{\frac{-\beta}{\alpha(\beta-6)}}\left(x-\left(1-\frac{\beta}{6}\right) t\right)\right)\right)
$$$$
-\frac{\frac{\sqrt{2}}{4}}{i \tanh \left(\frac{\sqrt{2}}{2} \sqrt{\frac{-\beta}{\alpha(\beta-6)}}\left(x-\left(1-\frac{\beta}{6}\right) t\right)\right) \pm \operatorname{sech}\left(\frac{\sqrt{2}}{2} \sqrt{\frac{-\beta}{\alpha(\beta-6)}}\left(x-\left(1-\frac{\beta}{6}\right) t\right)\right)},
$$

$$
\begin{aligned}
& u_{146,147}(x, t)=-\frac{1}{2}+ \\
& \frac{\sqrt{2}}{4}\left(i \tanh \left(\frac{\sqrt{2}}{2} \sqrt{\frac{-\beta}{\alpha(\beta-6)}}\left(x-\left(1-\frac{\beta}{6}\right) t\right)\right) \pm \operatorname{sech}\left(\frac{\sqrt{2}}{2} \sqrt{\frac{-\beta}{\alpha(\beta-6)}}\left(x-\left(1-\frac{\beta}{6}\right) t\right)\right)\right) \\
& +\frac{\frac{\sqrt{2}}{4}}{i \tanh \left(\frac{\sqrt{2}}{2} \sqrt{\frac{-\beta}{\alpha(\beta-6)}}\left(x-\left(1-\frac{\beta}{6}\right) t\right)\right) \mp \operatorname{sech}\left(\frac{\sqrt{2}}{2} \sqrt{\frac{-\beta}{\alpha(\beta-6)}}\left(x-\left(1-\frac{\beta}{6}\right) t\right)\right)},
\end{aligned}
$$




$$
\begin{aligned}
& u_{148,149}(x, t)=-\frac{1}{2}- \\
& \frac{\sqrt{2}}{4}\left(i \tanh \left(\frac{\sqrt{2}}{2} \sqrt{\frac{-\beta}{\alpha(\beta-6)}}\left(x-\left(1-\frac{\beta}{6}\right) t\right)\right) \pm \operatorname{sech}\left(\frac{\sqrt{2}}{2} \sqrt{\frac{-\beta}{\alpha(\beta-6)}}\left(x-\left(1-\frac{\beta}{6}\right) t\right)\right)\right) \\
& -\frac{\frac{\sqrt{2}}{4}}{i \tanh \left(\frac{\sqrt{2}}{2} \sqrt{\frac{-\beta}{\alpha(\beta-6)}}\left(x-\left(1-\frac{\beta}{6}\right) t\right)\right) \mp \operatorname{sech}\left(\frac{\sqrt{2}}{2} \sqrt{\frac{-\beta}{\alpha(\beta-6)}}\left(x-\left(1-\frac{\beta}{6}\right) t\right)\right)},
\end{aligned}
$$$$
\text { for } \frac{\beta}{\alpha(\beta-6)}>0 \text {, }
$$$$
u_{150,151}(x, t)=-\frac{1}{2}
$$$$
+\frac{1}{2} \tanh \left(\sqrt{\frac{\beta}{\alpha(\beta-6)}}\left(x-\left(1-\frac{\beta}{6}\right) t\right)\right) \pm \frac{i}{2} \operatorname{sech}\left(\sqrt{\frac{\beta}{\alpha(\beta-6)}}\left(x-\left(1-\frac{\beta}{6}\right) t\right)\right),
$$$$
u_{152,153}(x, t)=-\frac{1}{2}-
$$$$
\frac{1}{2} \tanh \left(\sqrt{\frac{\beta}{\alpha(\beta-6)}}\left(x-\left(1-\frac{\beta}{6}\right) t\right)\right) \pm \frac{i}{2} \operatorname{sech}\left(\sqrt{\frac{\beta}{\alpha(\beta-6)}}\left(x-\left(1-\frac{\beta}{6}\right) t\right)\right),
$$$$
u_{154,155}(x, t)=-\frac{1}{2}+
$$

$$
\frac{1}{2\left(\tanh \left(\sqrt{\frac{\beta}{\alpha(\beta-6)}}\left(x-\left(1-\frac{\beta}{6}\right) t\right)\right) \pm i \operatorname{sech}\left(\sqrt{\frac{\beta}{\alpha(\beta-6)}}\left(x-\left(1-\frac{\beta}{6}\right) t\right)\right)\right)}
$$$$
u_{156,157}(x, t)=-\frac{1}{2}-
$$

$$
\frac{1}{2\left(\tanh \left(\sqrt{\frac{\beta}{\alpha(\beta-6)}}\left(x-\left(1-\frac{\beta}{6}\right) t\right)\right) \pm i \operatorname{sech}\left(\sqrt{\frac{\beta}{\alpha(\beta-6)}}\left(x-\left(1-\frac{\beta}{6}\right) t\right)\right)\right)}
$$

$u_{158,159}(x, t)=-\frac{1}{2}-$

$$
\begin{gathered}
\quad \frac{1}{4}\left(\tanh \left(\frac{1}{2} \sqrt{\frac{\beta}{\alpha(\beta-6)}}\left(x-\left(1-\frac{\beta}{6}\right) t\right)\right) \pm i \operatorname{sech}\left(\frac{1}{2} \sqrt{\frac{\beta}{\alpha(\beta-6)}}\left(x-\left(1-\frac{\beta}{6}\right) t\right)\right)\right) \\
+\frac{\frac{1}{4}}{\tanh \left(\frac{1}{2} \sqrt{\frac{\beta}{\alpha(\beta-6)}}\left(x-\left(1-\frac{\beta}{6}\right) t\right)\right) \pm i \operatorname{sech}\left(\frac{1}{2} \sqrt{\frac{\beta}{\alpha(\beta-6)}}\left(x-\left(1-\frac{\beta}{6}\right) t\right)\right)},
\end{gathered}
$$

$u_{160,161}(x, t)=-\frac{1}{2}+$

$$
\frac{1}{4}\left(\tanh \left(\frac{1}{2} \sqrt{\frac{\beta}{\alpha(\beta-6)}}\left(x-\left(1-\frac{\beta}{6}\right) t\right)\right) \pm i \operatorname{sech}\left(\frac{1}{2} \sqrt{\frac{\beta}{\alpha(\beta-6)}}\left(x-\left(1-\frac{\beta}{6}\right) t\right)\right)\right)
$$$$
-\frac{\frac{1}{4}}{\tanh \left(\frac{1}{2} \sqrt{\frac{\beta}{\alpha(\beta-6)}}\left(x-\left(1-\frac{\beta}{6}\right) t\right)\right) \pm i \operatorname{sech}\left(\frac{1}{2} \sqrt{\frac{\beta}{\alpha(\beta-6)}}\left(x-\left(1-\frac{\beta}{6}\right) t\right)\right)},
$$ 


$$
\begin{aligned}
& u_{162,163}(x, t)=-\frac{1}{2}+ \\
& \frac{\sqrt{2}}{4}\left(\tan \left(\frac{\sqrt{2}}{2} \sqrt{\frac{\beta}{\alpha(\beta-6)}}\left(x-\left(1-\frac{\beta}{6}\right) t\right)\right) \pm \sec \left(\frac{\sqrt{2}}{2} \sqrt{\frac{\beta}{\alpha(\beta-6)}}\left(x-\left(1-\frac{\beta}{6}\right) t\right)\right)\right) \\
& +\frac{\frac{\sqrt{2}}{4}}{\tan \left(\frac{\sqrt{2}}{2} \sqrt{\frac{\beta}{\alpha(\beta-6)}}\left(x-\left(1-\frac{\beta}{6}\right) t\right)\right) \pm \sec \left(\frac{\sqrt{2}}{2} \sqrt{\frac{\beta}{\alpha(\beta-6)}}\left(x-\left(1-\frac{\beta}{6}\right) t\right)\right)}, \\
& u_{164,165}(x, t)=-\frac{1}{2}- \\
& \frac{\sqrt{2}}{4}\left(\tan \left(\frac{\sqrt{2}}{2} \sqrt{\frac{\beta}{\alpha(\beta-6)}}\left(x-\left(1-\frac{\beta}{6}\right) t\right)\right) \pm \sec \left(\frac{\sqrt{2}}{2} \sqrt{\frac{\beta}{\alpha(\beta-6)}}\left(x-\left(1-\frac{\beta}{6}\right) t\right)\right)\right) \\
& -\frac{\frac{\sqrt{2}}{4}}{\tan \left(\frac{\sqrt{2}}{2} \sqrt{\frac{\beta}{\alpha(\beta-6)}}\left(x-\left(1-\frac{\beta}{6}\right) t\right)\right) \pm \sec \left(\frac{\sqrt{2}}{2} \sqrt{\frac{\beta}{\alpha(\beta-6)}}\left(x-\left(1-\frac{\beta}{6}\right) t\right)\right)} \text {, } \\
& u_{166,167}(x, t)=-\frac{1}{2} \\
& +\frac{\sqrt{2}}{4}\left(\tan \left(\frac{\sqrt{2}}{2} \sqrt{\frac{\beta}{\alpha(\beta-6)}}\left(x-\left(1-\frac{\beta}{6}\right) t\right)\right) \pm \sec \left(\frac{\sqrt{2}}{2} \sqrt{\frac{\beta}{\alpha(\beta-6)}}\left(x-\left(1-\frac{\beta}{6}\right) t\right)\right)\right) \\
& +\frac{\frac{\sqrt{2}}{4}}{\tan \left(\frac{\sqrt{2}}{2} \sqrt{\frac{\beta}{\alpha(\beta-6)}}\left(x-\left(1-\frac{\beta}{6}\right) t\right)\right) \mp \sec \left(\frac{\sqrt{2}}{2} \sqrt{\frac{\beta}{\alpha(\beta-6)}}\left(x-\left(1-\frac{\beta}{6}\right) t\right)\right)} \text {, } \\
& u_{168,169}(x, t)=-\frac{1}{2}- \\
& \frac{\sqrt{2}}{4}\left(\tan \left(\frac{\sqrt{2}}{2} \sqrt{\frac{\beta}{\alpha(\beta-6)}}\left(x-\left(1-\frac{\beta}{6}\right) t\right)\right) \pm \sec \left(\frac{\sqrt{2}}{2} \sqrt{\frac{\beta}{\alpha(\beta-6)}}\left(x-\left(1-\frac{\beta}{6}\right) t\right)\right)\right) \\
& -\frac{\frac{\sqrt{2}}{4}}{\tan \left(\frac{\sqrt{2}}{2} \sqrt{\frac{\beta}{\alpha(\beta-6)}}\left(x-\left(1-\frac{\beta}{6}\right) t\right)\right) \mp \sec \left(\frac{\sqrt{2}}{2} \sqrt{\frac{\beta}{\alpha(\beta-6)}}\left(x-\left(1-\frac{\beta}{6}\right) t\right)\right)} \text {. }
\end{aligned}
$$

Using (9), the solution of (10) when $p=\frac{k^{2}-2}{2}, q=\frac{k^{2}}{2}, r=\frac{1}{4}$, and the sets of solutions (3)-(10), we get

$u_{170,171, \ldots, 177}(x, t)=a_{0}+a_{1} \frac{\operatorname{sn} \xi}{1 \pm \operatorname{dn} \xi}+b_{1} \frac{1 \pm \operatorname{dn} \xi}{\operatorname{sn} \xi}$, where $a_{0}, a_{1}$ and $b_{1}$ are defined in the sets of solutions (3)-(10).

Note that, when $k \rightarrow 1$ we obtain for $\frac{\beta}{\alpha(\beta-6)}>0$,

$$
u_{178,179}(x, t)=-\frac{1}{2}+\frac{\frac{1}{2} \tanh \left(\sqrt{\frac{\beta}{\alpha(\beta-6)}}\left(x-\left(1-\frac{\beta}{6}\right) t\right)\right)}{1 \pm \operatorname{sech}\left(\sqrt{\frac{\beta}{\alpha(\beta-6)}}\left(x-\left(1-\frac{\beta}{6}\right) t\right)\right)},
$$




$$
\begin{aligned}
& u_{180,181}(x, t)=-\frac{1}{2}-\frac{\frac{1}{2} \tanh \left(\sqrt{\frac{\beta}{\alpha(\beta-6)}}\left(x-\left(1-\frac{\beta}{6}\right) t\right)\right)}{1 \pm \operatorname{sech}\left(\sqrt{\frac{\beta}{\alpha(\beta-6)}}\left(x-\left(1-\frac{\beta}{6}\right) t\right)\right)}, \\
& u_{182,183}(x, t)=-\frac{1}{2}+\frac{\frac{1}{2}\left(1 \pm \operatorname{sech}\left(\sqrt{\frac{\beta}{\alpha(\beta-6)}}\left(x-\left(1-\frac{\beta}{6}\right) t\right)\right)\right)}{\tanh \left(\sqrt{\frac{\beta}{\alpha(\beta-6)}}\left(x-\left(1-\frac{\beta}{6}\right) t\right)\right)}, \\
& u_{184,185}(x, t)=-\frac{1}{2}-\frac{\frac{1}{2}\left(1 \pm \operatorname{sech}\left(\sqrt{\frac{\beta}{\alpha(\beta-6)}}\left(x-\left(1-\frac{\beta}{6}\right) t\right)\right)\right)}{\tanh \left(\sqrt{\frac{\beta}{\alpha(\beta-6)}}\left(x-\left(1-\frac{\beta}{6}\right) t\right)\right)}, \\
& u_{186,187}(x, t)=-\frac{1}{2}+\frac{\frac{\sqrt{2}}{4}\left(\tan \left(\frac{\sqrt{2}}{2} \sqrt{\frac{\beta}{\alpha(\beta-6)}}\left(x-\left(1-\frac{\beta}{6}\right) t\right)\right)\right)}{1 \pm \sec \left(\frac{\sqrt{2}}{2} \sqrt{\frac{\beta}{\alpha(\beta-6)}}\left(x-\left(1-\frac{\beta}{6}\right) t\right)\right)} \\
& +\frac{\frac{\sqrt{2}}{4}\left(1 \pm \sec \left(\frac{\sqrt{2}}{2} \sqrt{\frac{\beta}{\alpha(\beta-6)}}\left(x-\left(1-\frac{\beta}{6}\right) t\right)\right)\right)}{\tan \left(\frac{\sqrt{2}}{2} \sqrt{\frac{\beta}{\alpha(\beta-6)}}\left(x-\left(1-\frac{\beta}{6}\right) t\right)\right)}, \\
& u_{188,189}(x, t)=-\frac{1}{2}+\frac{\frac{\sqrt{2}}{4}\left(\tan \left(\frac{\sqrt{2}}{2} \sqrt{\frac{\beta}{\alpha(\beta-6)}}\left(x-\left(1-\frac{\beta}{6}\right) t\right)\right)\right)}{1 \pm \sec \left(\frac{\sqrt{2}}{2} \sqrt{\frac{\beta}{\alpha(\beta-6)}}\left(x-\left(1-\frac{\beta}{6}\right) t\right)\right)} \\
& +\frac{\frac{\sqrt{2}}{4}\left(1 \pm \sec \left(\frac{\sqrt{2}}{2} \sqrt{\frac{\beta}{\alpha(\beta-6)}}\left(x-\left(1-\frac{\beta}{6}\right) t\right)\right)\right)}{\tan \left(\frac{\sqrt{2}}{2} \sqrt{\frac{\beta}{\alpha(\beta-6)}}\left(x-\left(1-\frac{\beta}{6}\right) t\right)\right)}, \\
& u_{190,191}(x, t)=-\frac{1}{2}+\frac{\frac{1}{4}\left(\tanh \left(\frac{1}{2} \sqrt{\frac{\beta}{\alpha(\beta-6)}}\left(x-\left(1-\frac{\beta}{6}\right) t\right)\right)\right)}{1 \pm \operatorname{sech}\left(\frac{1}{2} \sqrt{\frac{\beta}{\alpha(\beta-6)}}\left(x-\left(1-\frac{\beta}{6}\right) t\right)\right)} \\
& +\frac{\frac{1}{4}\left(1 \pm \operatorname{sech}\left(\frac{1}{2} \sqrt{\frac{\beta}{\alpha(\beta-6)}}\left(x-\left(1-\frac{\beta}{6}\right) t\right)\right)\right)}{\tanh \left(\frac{1}{2} \sqrt{\frac{\beta}{\alpha(\beta-6)}}\left(x-\left(1-\frac{\beta}{6}\right) t\right)\right)}, \\
& u_{192,193}(x, t)=-\frac{1}{2}-\frac{\frac{1}{4}\left(\tanh \left(\frac{1}{2} \sqrt{\frac{\beta}{\alpha(\beta-6)}}\left(x-\left(1-\frac{\beta}{6}\right) t\right)\right)\right)}{1 \pm \operatorname{sech}\left(\frac{1}{2} \sqrt{\frac{\beta}{\alpha(\beta-6)}}\left(x-\left(1-\frac{\beta}{6}\right) t\right)\right)}
\end{aligned}
$$




$$
-\frac{\frac{1}{4}\left(1 \pm \operatorname{sech}\left(\frac{1}{2} \sqrt{\frac{\beta}{\alpha(\beta-6)}}\left(x-\left(1-\frac{\beta}{6}\right) t\right)\right)\right)}{\tanh \left(\frac{1}{2} \sqrt{\frac{\beta}{\alpha(\beta-6)}}\left(x-\left(1-\frac{\beta}{6}\right) t\right)\right)} .
$$

for $\frac{\beta}{\alpha(\beta-6)}<0$,

$$
\begin{aligned}
& u_{194,195}(x, t)=-\frac{1}{2}+i \frac{\frac{1}{2} \tan \left(\sqrt{\frac{-\beta}{\alpha(\beta-6)}}\left(x-\left(1-\frac{\beta}{6}\right) t\right)\right)}{1 \pm \sec \left(\sqrt{\frac{-\beta}{\alpha(\beta-6)}}\left(x-\left(1-\frac{\beta}{6}\right) t\right)\right)}, \\
& u_{196,197}(x, t)=-\frac{1}{2}-\frac{\frac{1}{2} \tan \left(\sqrt{\frac{-\beta}{\alpha(\beta-6)}}\left(x-\left(1-\frac{\beta}{6}\right) t\right)\right)}{1 \pm \sec \left(\sqrt{\frac{-\beta}{\alpha(\beta-6)}}\left(x-\left(1-\frac{\beta}{6}\right) t\right)\right)}, \\
& u_{198,199}(x, t)=-\frac{1}{2}+\frac{\frac{1}{2}\left(1 \pm \sec \left(\sqrt{\frac{-\beta}{\alpha(\beta-6)}}\left(x-\left(1-\frac{\beta}{6}\right) t\right)\right)\right)}{i \tan \left(\sqrt{\frac{-\beta}{\alpha(\beta-6)}}\left(x-\left(1-\frac{\beta}{6}\right) t\right)\right)} \text {, } \\
& u_{200,201}(x, t)=-\frac{1}{2}-\frac{\frac{1}{2}\left(1 \pm \sec \left(\sqrt{\frac{-\beta}{\alpha(\beta-6)}}\left(x-\left(1-\frac{\beta}{6}\right) t\right)\right)\right)}{i \tan \left(\sqrt{\frac{-\beta}{\alpha(\beta-6)}}\left(x-\left(1-\frac{\beta}{6}\right) t\right)\right)}, \\
& u_{202,203}(x, t)=-\frac{1}{2}+i \frac{\frac{\sqrt{2}}{4}\left(\tanh \left(\frac{\sqrt{2}}{2} \sqrt{\frac{-\beta}{\alpha(\beta-6)}}\left(x-\left(1-\frac{\beta}{6}\right) t\right)\right)\right)}{1 \pm \operatorname{sech}\left(\frac{\sqrt{2}}{2} \sqrt{\frac{-\beta}{\alpha(\beta-6)}}\left(x-\left(1-\frac{\beta}{6}\right) t\right)\right)} \\
& -i \frac{\frac{\sqrt{2}}{4}\left(1 \pm \operatorname{sech}\left(\frac{\sqrt{2}}{2} \sqrt{\frac{-\beta}{\alpha(\beta-6)}}\left(x-\left(1-\frac{\beta}{6}\right) t\right)\right)\right)}{\tanh \left(\frac{\sqrt{2}}{2} \sqrt{\frac{-\beta}{\alpha(\beta-6)}}\left(x-\left(1-\frac{\beta}{6}\right) t\right)\right)}, \\
& u_{204,205}(x, t)=-\frac{1}{2}-i \frac{\frac{\sqrt{2}}{4}\left(\tanh \left(\frac{\sqrt{2}}{2} \sqrt{\frac{-\beta}{\alpha(\beta-6)}}\left(x-\left(1-\frac{\beta}{6}\right) t\right)\right)\right)}{1 \pm \operatorname{sech}\left(\frac{\sqrt{2}}{2} \sqrt{\frac{-\beta}{\alpha(\beta-6)}}\left(x-\left(1-\frac{\beta}{6}\right) t\right)\right)} \\
& +i \frac{\frac{\sqrt{2}}{4}\left(1 \pm \operatorname{sech}\left(\frac{\sqrt{2}}{2} \sqrt{\frac{-\beta}{\alpha(\beta-6)}}\left(x-\left(1-\frac{\beta}{6}\right) t\right)\right)\right)}{\tanh \left(\frac{\sqrt{2}}{2} \sqrt{\frac{-\beta}{\alpha(\beta-6)}}\left(x-\left(1-\frac{\beta}{6}\right) t\right)\right)}, \\
& u_{206,207}(x, t)=-\frac{1}{2}+i \frac{\frac{1}{4}\left(\tan \left(\frac{1}{2} \sqrt{\frac{-\beta}{\alpha(\beta-6)}}\left(x-\left(1-\frac{\beta}{6}\right) t\right)\right)\right)}{1 \pm \sec \left(\frac{1}{2} \sqrt{\frac{-\beta}{\alpha(\beta-6)}}\left(x-\left(1-\frac{\beta}{6}\right) t\right)\right)}
\end{aligned}
$$




$$
\begin{aligned}
+ & i \frac{\frac{1}{4}\left(1+\sec \left(\frac{1}{2} \sqrt{\frac{-\beta}{\alpha(\beta-6)}}\left(x-\left(1-\frac{\beta}{6}\right) t\right)\right)\right)}{\tan \left(\frac{1}{2} \sqrt{\frac{-\beta}{\alpha(\beta-6)}}\left(x-\left(1-\frac{\beta}{6}\right) t\right)\right)}, \\
u_{208,209}(x, t)= & -\frac{1}{2}-i \frac{\frac{1}{4}\left(\tan \left(\frac{1}{2} \sqrt{\frac{-\beta}{\alpha(\beta-6)}}\left(x-\left(1-\frac{\beta}{6}\right) t\right)\right)\right)}{1 \pm \sec \left(\frac{1}{2} \sqrt{\frac{-\beta}{\alpha(\beta-6)}}\left(x-\left(1-\frac{\beta}{6}\right) t\right)\right)} \\
& -i \frac{\frac{1}{4}\left(1+\sec \left(\frac{1}{2} \sqrt{\frac{-\beta}{\alpha(\beta-6)}}\left(x-\left(1-\frac{\beta}{6}\right) t\right)\right)\right)}{\tan \left(\frac{1}{2} \sqrt{\frac{-\beta}{\alpha(\beta-6)}}\left(x-\left(1-\frac{\beta}{6}\right) t\right)\right)} .
\end{aligned}
$$

When $k \rightarrow 0$ we obtain, $\left[u_{31,32}(x, t)\right.$ and $\left.u_{33,34}(x, t)\right]$.

Using (9), the solution of (10) when $p=\frac{k^{2}+1}{2}, q=\frac{k^{2}-1}{2}, r=\frac{1-k^{2}}{4}$, and the sets of solutions $(3)-(10)$, we get

$u_{210,211, \ldots, 218}(x, t)=a_{0}+a_{1} \frac{\mathrm{dn} \xi}{1 \pm k \operatorname{sn} \xi}+b_{1} \frac{1 \pm k \mathrm{sn} \xi}{\mathrm{dn} \xi}$, where $a_{0}, a_{1}$ and $b_{1}$ are defined in the sets of solutions (3)-(10).

Note that, when $k \rightarrow 1$ we obtain constant solutions, also when $k \rightarrow 0$ we obtain constant solutions.

Using (9), the solution of (10) when $p=\frac{k^{2}+1}{2}, q=\frac{-1}{2}, r=\frac{-\left(1-k^{2}\right)^{2}}{4}$, and the sets of solutions (3)-(10), we get

$u_{219,220, \ldots, 226}(x, t)=a_{0}+a_{1}(k \operatorname{cn} \xi \pm \operatorname{dn} \xi)+\frac{b_{1}}{(k \operatorname{cn} \xi \pm \operatorname{dn} \xi)}$, where $a_{0}, a_{1}$ and $b_{1}$ are defined in the sets of solutions (3)-(10).

Note that, when $k \rightarrow 1$ we obtain, $\left[u_{3,4}(x, t)\right.$ and $\left.u_{5,6}(x, t)\right]$, when $k \rightarrow 0$ we obtain constant solution.

Using (9), the solution of (10) when $p=\frac{k^{2}+1}{2}, q=\frac{1-k^{2}}{2}, r=\frac{1-k^{2}}{4}$, and the sets of solutions (3)-(10), we get

$u_{227,228, \ldots, 234}(x, t)=a_{0}+a_{1}\left(\frac{\operatorname{cn} \xi}{1 \pm \operatorname{sn} \xi}\right)+b_{1} \frac{1 \pm \operatorname{sn} \xi}{\operatorname{cn} \xi}$, where $a_{0}, a_{1}$ and $b_{1}$ are defined in the sets of solutions (3)-(10).

Note that, when $k \rightarrow 1$ we obtain constant solution, when $k \rightarrow 0$ we obtain

$$
\begin{aligned}
& \text { for } \frac{\beta}{\alpha(\beta-6)}<0 \\
& \qquad u_{235,236}(x, t)=-\frac{1}{2}+\frac{i}{2} \frac{\cos \left(\sqrt{\frac{-\beta}{\alpha(\beta-6)}}\left(x-\left(1-\frac{\beta}{6}\right) t\right)\right)}{1 \pm \sin \left(\sqrt{\frac{-\beta}{\alpha(\beta-6)}}\left(x-\left(1-\frac{\beta}{6}\right) t\right)\right)},
\end{aligned}
$$




$$
\begin{aligned}
& u_{237,238}(x, t)=-\frac{1}{2}-\frac{i}{2} \frac{\cos \left(\sqrt{\frac{-\beta}{\alpha(\beta-6)}}\left(x-\left(1-\frac{\beta}{6}\right) t\right)\right)}{1 \pm \sin \left(\sqrt{\frac{-\beta}{\alpha(\beta-6)}}\left(x-\left(1-\frac{\beta}{6}\right) t\right)\right)}, \\
& u_{239,240}(x, t)=-\frac{1}{2}+\frac{i}{2} \frac{1 \pm \sin \left(\sqrt{\frac{-\beta}{\alpha(\beta-6)}}\left(x-\left(1-\frac{\beta}{6}\right) t\right)\right)}{\cos \left(\sqrt{\frac{-\beta}{\alpha(\beta-6)}}\left(x-\left(1-\frac{\beta}{6}\right) t\right)\right)}, \\
& u_{241,242}(x, t)=-\frac{1}{2}-\frac{i}{2} \frac{1 \pm \sin \left(\sqrt{\frac{-\beta}{\alpha(\beta-6)}}\left(x-\left(1-\frac{\beta}{6}\right) t\right)\right)}{\cos \left(\sqrt{\frac{-\beta}{\alpha(\beta-6)}}\left(x-\left(1-\frac{\beta}{6}\right) t\right)\right)}, \\
& u_{243,244}(x, t)=-\frac{1}{2}+\frac{i}{4} \frac{\cos \left(\frac{1}{2} \sqrt{\frac{-\beta}{\alpha(\beta-6)}}\left(x-\left(1-\frac{\beta}{6}\right) t\right)\right)}{1 \pm \sin \left(\frac{1}{2} \sqrt{\frac{-\beta}{\alpha(\beta-6)}}\left(x-\left(1-\frac{\beta}{6}\right) t\right)\right)} \\
& -\frac{i}{4} \frac{1 \pm \sin \left(\frac{1}{2} \sqrt{\frac{-\beta}{\alpha(\beta-6)}}\left(x-\left(1-\frac{\beta}{6}\right) t\right)\right)}{\cos \left(\frac{1}{2} \sqrt{\frac{-\beta}{\alpha(\beta-6)}}\left(x-\left(1-\frac{\beta}{6}\right) t\right)\right)}, \\
& u_{245,246}(x, t)=-\frac{1}{2}-\frac{i}{4} \frac{\cos \left(\frac{1}{2} \sqrt{\frac{-\beta}{\alpha(\beta-6)}}\left(x-\left(1-\frac{\beta}{6}\right) t\right)\right)}{1 \pm \sin \left(\frac{1}{2} \sqrt{\frac{-\beta}{\alpha(\beta-6)}}\left(x-\left(1-\frac{\beta}{6}\right) t\right)\right)} \\
& +\frac{i}{4} \frac{1 \pm \sin \left(\frac{1}{2} \sqrt{\frac{-\beta}{\alpha(\beta-6)}}\left(x-\left(1-\frac{\beta}{6}\right) t\right)\right)}{\cos \left(\frac{1}{2} \sqrt{\frac{-\beta}{\alpha(\beta-6)}}\left(x-\left(1-\frac{\beta}{6}\right) t\right)\right)}, \\
& u_{247,248}(x, t)=-\frac{1}{2}+\frac{i}{4} \frac{\cos \left(\frac{1}{2} \sqrt{\frac{-\beta}{\alpha(\beta-6)}}\left(x-\left(1-\frac{\beta}{6}\right) t\right)\right)}{1 \pm \sin \left(\frac{1}{2} \sqrt{\frac{-\beta}{\alpha(\beta-6)}}\left(x-\left(1-\frac{\beta}{6}\right) t\right)\right)} \\
& -\frac{i}{4} \frac{1 \mp \sin \left(\frac{1}{2} \sqrt{\frac{-\beta}{\alpha(\beta-6)}}\left(x-\left(1-\frac{\beta}{6}\right) t\right)\right)}{\cos \left(\frac{1}{2} \sqrt{\frac{-\beta}{\alpha(\beta-6)}}\left(x-\left(1-\frac{\beta}{6}\right) t\right)\right)} \text {, } \\
& u_{249,250}(x, t)=-\frac{1}{2}-\frac{i}{4} \frac{\cos \left(\frac{1}{2} \sqrt{\frac{-\beta}{\alpha(\beta-6)}}\left(x-\left(1-\frac{\beta}{6}\right) t\right)\right)}{1 \pm \sin \left(\frac{1}{2} \sqrt{\frac{-\beta}{\alpha(\beta-6)}}\left(x-\left(1-\frac{\beta}{6}\right) t\right)\right)}
\end{aligned}
$$




$$
\begin{aligned}
& +\frac{i}{4} \frac{1 \mp \sin \left(\frac{1}{2} \sqrt{\frac{-\beta}{\alpha(\beta-6)}}\left(x-\left(1-\frac{\beta}{6}\right) t\right)\right)}{\cos \left(\frac{1}{2} \sqrt{\frac{-\beta}{\alpha(\beta-6)}}\left(x-\left(1-\frac{\beta}{6}\right) t\right)\right)}, \\
u_{251,252}(x, t)= & -\frac{1}{2}+\frac{\sqrt{2}}{4} \frac{\cosh \left(\frac{\sqrt{2}}{2} \sqrt{\frac{-\beta}{\alpha(\beta-6)}}\left(x-\left(1-\frac{\beta}{6}\right) t\right)\right)}{1 \pm i \sinh \left(\frac{\sqrt{2}}{2} \sqrt{\frac{-\beta}{\alpha(\beta-6)}}\left(x-\left(1-\frac{\beta}{6}\right) t\right)\right)} \\
& -\frac{\sqrt{2}}{4} \frac{1 \mp i \sinh \left(\frac{\sqrt{2}}{2} \sqrt{\frac{-\beta}{\alpha(\beta-6)}}\left(x-\left(1-\frac{\beta}{6}\right) t\right)\right)}{\cosh \left(\frac{\sqrt{2}}{2} \sqrt{\left.\frac{-\beta}{\alpha(\beta-6)}\left(x-\left(1-\frac{\beta}{6}\right) t\right)\right)},\right.} \\
u_{253,254}(x, t)= & -\frac{1}{2}-\frac{\sqrt{2}}{4} \frac{\cosh \left(\frac{\sqrt{2}}{2} \sqrt{\frac{-\beta}{\alpha(\beta-6)}}\left(x-\left(1-\frac{\beta}{6}\right) t\right)\right)}{1 \pm i \sinh \left(\frac{\sqrt{2}}{2} \sqrt{\frac{-\beta}{\alpha(\beta-6)}}\left(x-\left(1-\frac{\beta}{6}\right) t\right)\right)} \\
& +\frac{\sqrt{2}}{4} \frac{1 \mp i \sinh \left(\frac{\sqrt{2}}{2} \sqrt{\frac{-\beta}{\alpha(\beta-6)}}\left(x-\left(1-\frac{\beta}{6}\right) t\right)\right)}{\cosh \left(\frac{\sqrt{2}}{2} \sqrt{\left.\frac{-\beta}{\alpha(\beta-6)}\left(x-\left(1-\frac{\beta}{6}\right) t\right)\right)} .\right.}
\end{aligned}
$$

for $\frac{\beta}{\alpha(\beta-6)}>0$,

$$
\begin{aligned}
& u_{255,256}(x, t)=-\frac{1}{2}+\frac{1}{2} \frac{\cosh \left(\sqrt{\frac{\beta}{\alpha(\beta-6)}}\left(x-\left(1-\frac{\beta}{6}\right) t\right)\right)}{i \pm \sinh \left(\sqrt{\frac{\beta}{\alpha(\beta-6)}}\left(x-\left(1-\frac{\beta}{6}\right) t\right)\right)}, \\
& u_{257,258}(x, t)=-\frac{1}{2}-\frac{1}{2} \frac{\cosh \left(\sqrt{\frac{\beta}{\alpha(\beta-6)}}\left(x-\left(1-\frac{\beta}{6}\right) t\right)\right)}{i \pm \sinh \left(\sqrt{\frac{\beta}{\alpha(\beta-6)}}\left(x-\left(1-\frac{\beta}{6}\right) t\right)\right)}, \\
& u_{259,260}(x, t)=-\frac{1}{2}+\frac{1}{2} \frac{i \pm \sinh \left(\sqrt{\frac{\beta}{\alpha(\beta-6)}}\left(x-\left(1-\frac{\beta}{6}\right) t\right)\right)}{\cosh \left(\sqrt{\frac{\beta}{\alpha(\beta-6)}}\left(x-\left(1-\frac{\beta}{6}\right) t\right)\right)}, \\
& u_{261,262}(x, t)=-\frac{1}{2}-\frac{1}{2} \frac{i \pm \sinh \left(\sqrt{\frac{\beta}{\alpha(\beta-6)}}\left(x-\left(1-\frac{\beta}{6}\right) t\right)\right)}{\cosh \left(\sqrt{\frac{\beta}{\alpha(\beta-6)}}\left(x-\left(1-\frac{\beta}{6}\right) t\right)\right)}, \\
& u_{263,264}(x, t)=-\frac{1}{2}+\frac{i}{4} \frac{\cosh \left(\frac{1}{2} \sqrt{\frac{\beta}{\alpha(\beta-6)}}\left(x-\left(1-\frac{\beta}{6}\right) t\right)\right)}{1 \pm i \sinh \left(\frac{1}{2} \sqrt{\frac{\beta}{\alpha(\beta-6)}}\left(x-\left(1-\frac{\beta}{6}\right) t\right)\right)}
\end{aligned}
$$




$$
\begin{aligned}
& -\frac{i}{4} \frac{1 \pm i \sinh \left(\frac{1}{2} \sqrt{\frac{\beta}{\alpha(\beta-6)}}\left(x-\left(1-\frac{\beta}{6}\right) t\right)\right)}{\cosh \left(\frac{1}{2} \sqrt{\frac{\beta}{\alpha(\beta-6)}}\left(x-\left(1-\frac{\beta}{6}\right) t\right)\right)} \\
& u_{265,266}(x, t)=-\frac{1}{2}-\frac{i}{4} \frac{\cosh \left(\frac{1}{2} \sqrt{\frac{\beta}{\alpha(\beta-6)}}\left(x-\left(1-\frac{\beta}{6}\right) t\right)\right)}{1 \pm i \sinh \left(\frac{1}{2} \sqrt{\frac{\beta}{\alpha(\beta-6)}}\left(x-\left(1-\frac{\beta}{6}\right) t\right)\right)} \\
& +\frac{i}{4} \frac{1 \pm i \sinh \left(\frac{1}{2} \sqrt{\frac{\beta}{\alpha(\beta-6)}}\left(x-\left(1-\frac{\beta}{6}\right) t\right)\right)}{\cosh \left(\frac{1}{2} \sqrt{\frac{\beta}{\alpha(\beta-6)}}\left(x-\left(1-\frac{\beta}{6}\right) t\right)\right)} \text {, } \\
& u_{267,268}(x, t)=-\frac{1}{2}+\frac{i}{4} \frac{\cosh \left(\frac{1}{2} \sqrt{\frac{\beta}{\alpha(\beta-6)}}\left(x-\left(1-\frac{\beta}{6}\right) t\right)\right)}{1 \pm i \sinh \left(\frac{1}{2} \sqrt{\frac{\beta}{\alpha(\beta-6)}}\left(x-\left(1-\frac{\beta}{6}\right) t\right)\right)} \\
& -\frac{i}{4} \frac{1 \mp i \sinh \left(\frac{1}{2} \sqrt{\frac{\beta}{\alpha(\beta-6)}}\left(x-\left(1-\frac{\beta}{6}\right) t\right)\right)}{\cosh \left(\frac{1}{2} \sqrt{\frac{\beta}{\alpha(\beta-6)}}\left(x-\left(1-\frac{\beta}{6}\right) t\right)\right)} \text {, } \\
& u_{269,270}(x, t)=-\frac{1}{2}-\frac{i}{4} \frac{\cosh \left(\frac{1}{2} \sqrt{\frac{\beta}{\alpha(\beta-6)}}\left(x-\left(1-\frac{\beta}{6}\right) t\right)\right)}{1 \pm i \sinh \left(\frac{1}{2} \sqrt{\frac{\beta}{\alpha(\beta-6)}}\left(x-\left(1-\frac{\beta}{6}\right) t\right)\right)} \\
& +\frac{i}{4} \frac{1 \mp i \sinh \left(\frac{1}{2} \sqrt{\frac{\beta}{\alpha(\beta-6)}}\left(x-\left(1-\frac{\beta}{6}\right) t\right)\right)}{\cosh \left(\frac{1}{2} \sqrt{\frac{\beta}{\alpha(\beta-6)}}\left(x-\left(1-\frac{\beta}{6}\right) t\right)\right)} \text {, } \\
& u_{271,272}(x, t)=-\frac{1}{2}+\frac{\sqrt{2}}{4} \frac{\cos \left(\frac{\sqrt{2}}{2} \sqrt{\frac{\beta}{\alpha(\beta-6)}}\left(x-\left(1-\frac{\beta}{6}\right) t\right)\right)}{1 \pm \sin \left(\frac{\sqrt{2}}{2} \sqrt{\frac{\beta}{\alpha(\beta-6)}}\left(x-\left(1-\frac{\beta}{6}\right) t\right)\right)} \\
& -\frac{\sqrt{2}}{4} \frac{1 \mp \sin \left(\frac{\sqrt{2}}{2} \sqrt{\frac{\beta}{\alpha(\beta-6)}}\left(x-\left(1-\frac{\beta}{6}\right) t\right)\right)}{\cos \left(\frac{\sqrt{2}}{2} \sqrt{\frac{\beta}{\alpha(\beta-6)}}\left(x-\left(1-\frac{\beta}{6}\right) t\right)\right)} \\
& u_{273,274}(x, t)=-\frac{1}{2}-\frac{\sqrt{2}}{4} \frac{\cos \left(\frac{\sqrt{2}}{2} \sqrt{\frac{\beta}{\alpha(\beta-6)}}\left(x-\left(1-\frac{\beta}{6}\right) t\right)\right)}{1 \pm \sin \left(\frac{\sqrt{2}}{2} \sqrt{\frac{\beta}{\alpha(\beta-6)}}\left(x-\left(1-\frac{\beta}{6}\right) t\right)\right)}
\end{aligned}
$$




$$
+\frac{\sqrt{2}}{4} \frac{1 \mp \sin \left(\frac{\sqrt{2}}{2} \sqrt{\frac{\beta}{\alpha(\beta-6)}}\left(x-\left(1-\frac{\beta}{6}\right) t\right)\right)}{\cos \left(\frac{\sqrt{2}}{2} \sqrt{\frac{\beta}{\alpha(\beta-6)}}\left(x-\left(1-\frac{\beta}{6}\right) t\right)\right)} .
$$

Using (9), the solution of (10) when $p=\frac{1-2 k^{2}}{2}, q=\frac{1}{2}, r=\frac{k^{2}}{4}$, and the sets of solutions (3)-(10), we get

$u_{275,276, \ldots 282}(x, t)=a_{0}+a_{1}(k \operatorname{sn} \xi \pm i \operatorname{dn} \xi)+b_{1} \frac{1}{k \operatorname{sn} \xi \pm i \operatorname{dn} \xi}$, where $a_{0}, a_{1}$ and $b_{1}$ are defined in the sets of solutions (3)-(10).

Note that, when $k \rightarrow 1$ we obtain, $\left[u_{130,131}(x, t), u_{132,133}(x, t), \ldots, u_{168,169}\right.$ $(x, t)]$, when $k \rightarrow 0$, we obtain constant solutions.

Using (9), the solution of (10) when $p=\frac{k^{2}-2}{2}, q=\frac{k^{2}}{2}, r=\frac{k^{2}}{4}$, and the sets of solutions (3)-(10), we get

$u_{283,284, \ldots, 290}(x, t)=a_{0}+a_{1}(k \operatorname{sn} \xi \pm i \operatorname{cn} \xi)+b_{1} \frac{1}{k \operatorname{sn} \xi \pm i \operatorname{cn} \xi}$, where $a_{0}, a_{1}$ and $b_{1}$ are defined in the sets of solutions (3)-(10).

Note that, when $k \rightarrow 1$ we obtain, $\left[u_{130,131}(x, t), u_{132,133}(x, t), \ldots, u_{168,169}\right.$ $(x, t)]$, when $k \rightarrow 0$, we obtain constant solutions.

Using (9), the solution of (10) when $p=\frac{k^{2}-2}{2}, q=\frac{1}{2}, r=\frac{k^{4}}{4}$, and the sets of solutions (3)-(10), we get

$u_{291,292, \ldots, 298}(x, t)=a_{0}+a_{1}(\mathrm{~ns} \xi \pm \mathrm{ds} \xi)+b_{1} \frac{1}{\mathrm{~ns} \xi \pm \mathrm{ds} \xi}$, where $a_{0}, a_{1}$ and $b_{1}$ are defined in the sets of solutions (3)-(10).

Note that, when $k \rightarrow 1$ we obtain, for $\frac{\beta}{\alpha(\beta-6)}>0$,

$$
\begin{aligned}
& u_{299,300}(x, t)=-\frac{1}{2}+ \\
& \frac{1}{2}\left(\operatorname{coth}\left(\sqrt{\frac{\beta}{\alpha(\beta-6)}}\left(x-\left(1-\frac{\beta}{6}\right) t\right)\right) \pm \operatorname{csch}\left(\sqrt{\frac{\beta}{\alpha(\beta-6)}}\left(x-\left(1-\frac{\beta}{6}\right) t\right)\right)\right), \\
& u_{301,302}(x, t)=-\frac{1}{2}- \\
& \frac{1}{2}\left(\operatorname{coth}\left(\sqrt{\frac{\beta}{\alpha(\beta-6)}}\left(x-\left(1-\frac{\beta}{6}\right) t\right)\right) \pm \operatorname{csch}\left(\sqrt{\frac{\beta}{\alpha(\beta-6)}}\left(x-\left(1-\frac{\beta}{6}\right) t\right)\right)\right), \\
& u_{303,304}(x, t)=-\frac{1}{2}+
\end{aligned}
$$

$$
\begin{aligned}
& \frac{1}{2\left(\operatorname{coth}\left(\sqrt{\frac{\beta}{\alpha(\beta-6)}}\left(x-\left(1-\frac{\beta}{6}\right) t\right)\right) \pm \operatorname{csch}\left(\sqrt{\frac{\beta}{\alpha(\beta-6)}}\left(x-\left(1-\frac{\beta}{6}\right) t\right)\right)\right)}, \\
& u_{305,306}(x, t)=-\frac{1}{2}- \\
& \frac{1}{2\left(\operatorname{coth}\left(\sqrt{\frac{\beta}{\alpha(\beta-6)}}\left(x-\left(1-\frac{\beta}{6}\right) t\right)\right) \pm \operatorname{csch}\left(\sqrt{\frac{\beta}{\alpha(\beta-6)}}\left(x-\left(1-\frac{\beta}{6}\right) t\right)\right)\right)},
\end{aligned}
$$




$$
\begin{aligned}
& u_{307,308}(x, t)=-\frac{1}{2}+ \\
& \frac{\sqrt{2}}{4}\left(\cot \left(\frac{\sqrt{2}}{2} \sqrt{\frac{\beta}{\alpha(\beta-6)}}\left(x-\left(1-\frac{\beta}{6}\right) t\right)\right) \pm \csc \left(\frac{\sqrt{2}}{2} \sqrt{\frac{\beta}{\alpha(\beta-6)}}\left(x-\left(1-\frac{\beta}{6}\right) t\right)\right)\right) \\
& \frac{+\sqrt{2}}{4\left(\cot \left(\frac{\sqrt{2}}{2} \sqrt{\frac{\beta}{\alpha(\beta-6)}}\left(x-\left(1-\frac{\beta}{6}\right) t\right)\right) \pm \csc \left(\frac{\sqrt{2}}{2} \sqrt{\frac{\beta}{\alpha(\beta-6)}}\left(x-\left(1-\frac{\beta}{6}\right) t\right)\right)\right)}
\end{aligned}
$$

$u_{309,310}(x, t)=-\frac{1}{2}+$

$$
\begin{aligned}
& \frac{\sqrt{2}}{4}\left(\cot \left(\frac{\sqrt{2}}{2} \sqrt{\frac{\beta}{\alpha(\beta-6)}}\left(x-\left(1-\frac{\beta}{6}\right) t\right)\right) \pm \csc \left(\frac{\sqrt{2}}{2} \sqrt{\frac{\beta}{\alpha(\beta-6)}}\left(x-\left(1-\frac{\beta}{6}\right) t\right)\right)\right) \\
& 4\left(\cot \left(\frac{\sqrt{2}}{2} \sqrt{\frac{\beta}{\alpha(\beta-6)}}\left(x-\left(1-\frac{\beta}{6}\right) t\right)\right) \mp \csc \left(\frac{\sqrt{2}}{2} \sqrt{\frac{\beta}{\alpha(\beta-6)}}\left(x-\left(1-\frac{\beta}{6}\right) t\right)\right)\right)
\end{aligned}
$$$$
\begin{aligned}
& u_{311,312}(x, t)=-\frac{1}{2}- \\
& \frac{\sqrt{2}}{4}\left(\cot \left(\frac{\sqrt{2}}{2} \sqrt{\frac{\beta}{\alpha(\beta-6)}}\left(x-\left(1-\frac{\beta}{6}\right) t\right)\right) \pm \csc \left(\frac{\sqrt{2}}{2} \sqrt{\frac{\beta}{\alpha(\beta-6)}}\left(x-\left(1-\frac{\beta}{6}\right) t\right)\right)\right)
\end{aligned}
$$$$
\frac{-\sqrt{2}}{4\left(\cot \left(\frac{\sqrt{2}}{2} \sqrt{\frac{\beta}{\alpha(\beta-6)}}\left(x-\left(1-\frac{\beta}{6}\right) t\right)\right) \pm \csc \left(\frac{\sqrt{2}}{2} \sqrt{\frac{\beta}{\alpha(\beta-6)}}\left(x-\left(1-\frac{\beta}{6}\right) t\right)\right)\right)},
$$

$u_{313,314}(x, t)=-\frac{1}{2}-$

$$
\begin{aligned}
& \frac{\sqrt{2}}{4}\left(\cot \left(\frac{\sqrt{2}}{2} \sqrt{\frac{\beta}{\alpha(\beta-6)}}\left(x-\left(1-\frac{\beta}{6}\right) t\right)\right) \pm \csc \left(\frac{\sqrt{2}}{2} \sqrt{\frac{\beta}{\alpha(\beta-6)}}\left(x-\left(1-\frac{\beta}{6}\right) t\right)\right)\right) \\
& \frac{-\sqrt{2}}{4\left(\cot \left(\frac{\sqrt{2}}{2} \sqrt{\frac{\beta}{\alpha(\beta-6)}}\left(x-\left(1-\frac{\beta}{6}\right) t\right)\right) \mp \csc \left(\frac{\sqrt{2}}{2} \sqrt{\frac{\beta}{\alpha(\beta-6)}}\left(x-\left(1-\frac{\beta}{6}\right) t\right)\right)\right)},
\end{aligned}
$$

$u_{315,316}(x, t)=-\frac{1}{2}+$

$\frac{1}{4}\left(\operatorname{coth}\left(\frac{1}{2} \sqrt{\frac{\beta}{\alpha(\beta-6)}}\left(x-\left(1-\frac{\beta}{6}\right) t\right)\right) \pm \operatorname{csch}\left(\frac{1}{2} \sqrt{\frac{\beta}{\alpha(\beta-6)}}\left(x-\left(1-\frac{\beta}{6}\right) t\right)\right)\right)$

$$
\frac{-1}{4\left(\operatorname{coth}\left(\frac{1}{2} \sqrt{\frac{\beta}{\alpha(\beta-6)}}\left(x-\left(1-\frac{\beta}{6}\right) t\right)\right) \mp \operatorname{csch}\left(\frac{1}{2} \sqrt{\frac{\beta}{\alpha(\beta-6)}}\left(x-\left(1-\frac{\beta}{6}\right) t\right)\right)\right)},
$$

$u_{317,318}(x, t)=-\frac{1}{2}-$ 
$\frac{1}{4}\left(\operatorname{coth}\left(\frac{1}{2} \sqrt{\frac{\beta}{\alpha(\beta-6)}}\left(x-\left(1-\frac{\beta}{6}\right) t\right)\right) \pm \operatorname{csch}\left(\frac{1}{2} \sqrt{\frac{\beta}{\alpha(\beta-6)}}\left(x-\left(1-\frac{\beta}{6}\right) t\right)\right)\right)$ $\frac{+1}{4\left(\operatorname{coth}\left(\frac{1}{2} \sqrt{\frac{\beta}{\alpha(\beta-6)}}\left(x-\left(1-\frac{\beta}{6}\right) t\right)\right) \mp \operatorname{csch}\left(\frac{1}{2} \sqrt{\frac{\beta}{\alpha(\beta-6)}}\left(x-\left(1-\frac{\beta}{6}\right) t\right)\right)\right)}$, for $\frac{\beta}{\alpha(\beta-6)}<0$, $u_{319,320}(x, t)=-\frac{1}{2}+$ $\frac{i}{2}\left(\cot \left(\sqrt{\frac{-\beta}{\alpha(\beta-6)}}\left(x-\left(1-\frac{\beta}{6}\right) t\right)\right) \pm \csc \left(\sqrt{\frac{-\beta}{\alpha(\beta-6)}}\left(x-\left(1-\frac{\beta}{6}\right) t\right)\right)\right)$, $u_{321,322}(x, t)=-\frac{1}{2}-$

$\frac{i}{2}\left(\cot \left(\sqrt{\frac{-\beta}{\alpha(\beta-6)}}\left(x-\left(1-\frac{\beta}{6}\right) t\right)\right) \pm \csc \left(\sqrt{\frac{-\beta}{\alpha(\beta-6)}}\left(x-\left(1-\frac{\beta}{6}\right) t\right)\right)\right)$, $u_{323,324}(x, t)=-\frac{1}{2}+$

$$
\frac{i}{2\left(\cot \left(\sqrt{\frac{-\beta}{\alpha(\beta-6)}}\left(x-\left(1-\frac{\beta}{6}\right) t\right)\right) \pm \csc \left(\sqrt{\frac{-\beta}{\alpha(\beta-6)}}\left(x-\left(1-\frac{\beta}{6}\right) t\right)\right)\right)},
$$

$u_{325,326}(x, t)=-\frac{1}{2}-$

$$
\frac{i}{2\left(\cot \left(\sqrt{\frac{-\beta}{\alpha(\beta-6)}}\left(x-\left(1-\frac{\beta}{6}\right) t\right)\right) \pm \csc \left(\sqrt{\frac{-\beta}{\alpha(\beta-6)}}\left(x-\left(1-\frac{\beta}{6}\right) t\right)\right)\right)},
$$

$u_{327,328}(x, t)=-\frac{1}{2}$

$+\frac{i \sqrt{2}}{4}\left(\operatorname{coth}\left(\frac{\sqrt{2}}{2} \sqrt{\frac{-\beta}{\alpha(\beta-6)}}\left(x-\left(1-\frac{\beta}{6}\right) t\right)\right) \pm \operatorname{csch}\left(\frac{\sqrt{2}}{2} \sqrt{\frac{-\beta}{\alpha(\beta-6)}}\left(x-\left(1-\frac{\beta}{6}\right) t\right)\right)\right)$ $\frac{-i \sqrt{2}}{4\left(\operatorname{coth}\left(\frac{\sqrt{2}}{2} \sqrt{\frac{-\beta}{\alpha(\beta-6)}}\left(x-\left(1-\frac{\beta}{6}\right) t\right)\right) \pm \operatorname{csch}\left(\frac{\sqrt{2}}{2} \sqrt{\frac{-\beta}{\alpha(\beta-6)}}\left(x-\left(1-\frac{\beta}{6}\right) t\right)\right)\right)}$, $u_{329,330}(x, t)=-\frac{1}{2}$ $+\frac{i \sqrt{2}}{4}\left(\operatorname{coth}\left(\frac{\sqrt{2}}{2} \sqrt{\frac{-\beta}{\alpha(\beta-6)}}\left(x-\left(1-\frac{\beta}{6}\right) t\right)\right) \pm \operatorname{csch}\left(\frac{\sqrt{2}}{2} \sqrt{\frac{-\beta}{\alpha(\beta-6)}}\left(x-\left(1-\frac{\beta}{6}\right) t\right)\right)\right)$ $\frac{-i \sqrt{2}}{4\left(\operatorname{coth}\left(\frac{\sqrt{2}}{2} \sqrt{\frac{-\beta}{\alpha(\beta-6)}}\left(x-\left(1-\frac{\beta}{6}\right) t\right)\right) \mp \operatorname{csch}\left(\frac{\sqrt{2}}{2} \sqrt{\frac{-\beta}{\alpha(\beta-6)}}\left(x-\left(1-\frac{\beta}{6}\right) t\right)\right)\right)}$, $u_{331,332}(x, t)=-\frac{1}{2}$ 


$$
\begin{aligned}
& -\frac{i \sqrt{2}}{4}\left(\operatorname{coth}\left(\frac{\sqrt{2}}{2} \sqrt{\frac{-\beta}{\alpha(\beta-6)}}\left(x-\left(1-\frac{\beta}{6}\right) t\right)\right) \pm \operatorname{csch}\left(\frac{\sqrt{2}}{2} \sqrt{\frac{-\beta}{\alpha(\beta-6)}}\left(x-\left(1-\frac{\beta}{6}\right) t\right)\right)\right) \\
& \frac{+i \sqrt{2}}{4\left(\operatorname{coth}\left(\frac{\sqrt{2}}{2} \sqrt{\frac{-\beta}{\alpha(\beta-6)}}\left(x-\left(1-\frac{\beta}{6}\right) t\right)\right) \pm \operatorname{csch}\left(\frac{\sqrt{2}}{2} \sqrt{\frac{-\beta}{\alpha(\beta-6)}}\left(x-\left(1-\frac{\beta}{6}\right) t\right)\right)\right)} \\
& u_{333,334}(x, t)=-\frac{1}{2} \\
& -\frac{i \sqrt{2}}{4}\left(\operatorname{coth}\left(\frac{\sqrt{2}}{2} \sqrt{\frac{-\beta}{\alpha(\beta-6)}}\left(x-\left(1-\frac{\beta}{6}\right) t\right)\right) \pm \operatorname{csch}\left(\frac{\sqrt{2}}{2} \sqrt{\frac{-\beta}{\alpha(\beta-6)}}\left(x-\left(1-\frac{\beta}{6}\right) t\right)\right)\right) \\
& \frac{+i \sqrt{2}}{4\left(\operatorname{coth}\left(\frac{\sqrt{2}}{2} \sqrt{\frac{-\beta}{\alpha(\beta-6)}}\left(x-\left(1-\frac{\beta}{6}\right) t\right)\right) \mp \operatorname{csch}\left(\frac{\sqrt{2}}{2} \sqrt{\frac{-\beta}{\alpha(\beta-6)}}\left(x-\left(1-\frac{\beta}{6}\right) t\right)\right)\right)},
\end{aligned}
$$$$
u_{335,336}(x, t)=-\frac{1}{2}+
$$

$$
\begin{aligned}
& \frac{i}{4}\left(\cot \left(\frac{1}{2} \sqrt{\frac{-\beta}{\alpha(\beta-6)}}\left(x-\left(1-\frac{\beta}{6}\right) t\right)\right) \pm \csc \left(\frac{1}{2} \sqrt{\frac{-\beta}{\alpha(\beta-6)}}\left(x-\left(1-\frac{\beta}{6}\right) t\right)\right)\right) \\
& \frac{+i}{4\left(\cot \left(\frac{1}{2} \sqrt{\frac{-\beta}{\alpha(\beta-6)}}\left(x-\left(1-\frac{\beta}{6}\right) t\right)\right) \mp \csc \left(\frac{1}{2} \sqrt{\frac{-\beta}{\alpha(\beta-6)}}\left(x-\left(1-\frac{\beta}{6}\right) t\right)\right)\right)}
\end{aligned}
$$

$u_{337,338}(x, t)=-\frac{1}{2}-$

$$
\begin{gathered}
\frac{i}{4}\left(\cot \left(\frac{1}{2} \sqrt{\frac{-\beta}{\alpha(\beta-6)}}\left(x-\left(1-\frac{\beta}{6}\right) t\right)\right) \pm \csc \left(\frac{1}{2} \sqrt{\frac{-\beta}{\alpha(\beta-6)}}\left(x-\left(1-\frac{\beta}{6}\right) t\right)\right)\right) \\
\frac{-i}{4\left(\cot \left(\frac{1}{2} \sqrt{\frac{-\beta}{\alpha(\beta-6)}}\left(x-\left(1-\frac{\beta}{6}\right) t\right)\right) \mp \csc \left(\frac{1}{2} \sqrt{\frac{-\beta}{\alpha(\beta-6)}}\left(x-\left(1-\frac{\beta}{6}\right) t\right)\right)\right)} .
\end{gathered}
$$

When $k \rightarrow 0$, we obtain, $\left[u_{31,32}(x, t)\right.$ and $\left.u_{33,34}(x, t)\right]$.

Using (9), the solution of (10) when $p=\frac{1-2 k^{2}}{2}, q=\frac{1}{2}, r=\frac{1}{4}$, and the sets of solutions (3)-(10), we get

$u_{339,340, \ldots, 346}(x, t)=a_{0}+a_{1}(\mathrm{~ns} \xi-\operatorname{cs} \xi)+b_{1} \frac{1}{\operatorname{ns} \xi-\operatorname{cs} \xi}$, where $a_{0}, a_{1}$ and $b_{1}$ are defined in the sets of solutions (3)-(10).

Note that, when $k \rightarrow 1$ we obtain, $\left[u_{299,300}(x, t), u_{301,302}(x, t), \ldots, u_{337,338}(x, t)\right]$, also when $k \rightarrow 0$ we obtain, $\left[u_{299,300}(x, t), u_{301,302}(x, t), \ldots, u_{337,338}(x, t)\right]$.

Using (9), the solution of (10) when $p=\frac{1-2 k^{2}}{2}, q=\frac{1}{2}, r=\frac{1}{4}$, and the sets of solutions (3)-(10), we get

$u_{347,348, \ldots, 354}(x, t)=a_{0}+a_{1}\left(\frac{\operatorname{cn} \xi}{\sqrt{1-k^{2}} \operatorname{sn} \xi \pm \operatorname{dn} \xi}\right)+b_{1} \frac{\sqrt{1-k^{2}} \operatorname{sn} \xi \pm \operatorname{dn} \xi}{\operatorname{cn} \xi}$, where $a_{0}, a_{1}$ and $b_{1}$ are defined in the sets of solutions (3)-(10). 
Note that, when $k \rightarrow 1$ constant solutions, when $k \rightarrow 0$ we obtain, $\left[u_{235,236}(x, t)\right.$, $\left.u_{237,238}(x, t), \ldots, u_{273,274}(x, t)\right]$.

Using (9), the solution of (10) when $p=\frac{1+k^{2}}{2}, q=\frac{\left(1-k^{2}\right)^{2}}{2}, r=\frac{1}{4}, \quad$ and the sets of solutions (3)-(10), we get

$u_{355,356, \ldots, 362}(x, t)=a_{0}+a_{1}\left(\frac{\operatorname{sn} \xi}{\operatorname{cn} \xi \pm \operatorname{dn} \xi}\right)+b_{1} \frac{\operatorname{cn} \xi \pm \operatorname{dn} \xi}{\operatorname{sn} \xi}$, where $a_{0}, a_{1}$ and $b_{1}$ are defined in the sets of solutions (3)-(10).

Note that, when $k \rightarrow 1$ we get $\left[u_{31,32}(x, t)\right.$ and $\left.u_{33,34}(x, t)\right]$, when $k \rightarrow 0$ we obtain for $\frac{\beta}{\alpha(\beta-6)}>0$,

$$
\begin{aligned}
& u_{363,364}(x, t)=-\frac{1}{2}+\frac{i}{2}\left(\frac{\sin \left(\sqrt{\frac{\beta}{\alpha(\beta-6)}}\left(x-\left(1-\frac{\beta}{6}\right) t\right)\right)}{\cos \left(\sqrt{\frac{\beta}{\alpha(\beta-6)}}\left(x-\left(1-\frac{\beta}{6}\right) t\right)\right) \pm 1}\right), \\
& u_{365,366}(x, t)=-\frac{1}{2}-\frac{i}{2}\left(\frac{\sin \left(\sqrt{\frac{\beta}{\alpha(\beta-6)}}\left(x-\left(1-\frac{\beta}{6}\right) t\right)\right)}{\cos \left(\sqrt{\frac{\beta}{\alpha(\beta-6)}}\left(x-\left(1-\frac{\beta}{6}\right) t\right)\right) \pm 1}\right), \\
& u_{367,368}(x, t)=-\frac{1}{2}+\frac{i}{2}\left(\frac{\cos \left(\sqrt{\frac{\beta}{\alpha(\beta-6)}}\left(x-\left(1-\frac{\beta}{6}\right) t\right)\right) \pm 1}{\sin \left(\sqrt{\frac{\beta}{\alpha(\beta-6)}}\left(x-\left(1-\frac{\beta}{6}\right) t\right)\right)}\right), \\
& u_{369,370}(x, t)=-\frac{1}{2}-\frac{i}{2}\left(\frac{\cos \left(\sqrt{\frac{\beta}{\alpha(\beta-6)}}\left(x-\left(1-\frac{\beta}{6}\right) t\right)\right) \pm 1}{\sin \left(\sqrt{\frac{\beta}{\alpha(\beta-6)}}\left(x-\left(1-\frac{\beta}{6}\right) t\right)\right)}\right), \\
& u_{371,372}(x, t)=-\frac{1}{2}+\frac{1}{4} \frac{\cosh \left(\frac{1}{2} \sqrt{\frac{\beta}{\alpha(\beta-6)}}\left(x-\left(1-\frac{\beta}{6}\right) t\right)\right) \pm 1}{\sinh \left(\frac{1}{2} \sqrt{\frac{\beta}{\alpha(\beta-6)}}\left(x-\left(1-\frac{\beta}{6}\right) t\right)\right)} \\
& +\frac{1}{4} \frac{\sinh \left(\frac{1}{2} \sqrt{\frac{\beta}{\alpha(\beta-6)}}\left(x-\left(1-\frac{\beta}{6}\right) t\right)\right)}{\cosh \left(\frac{1}{2} \sqrt{\frac{\beta}{\alpha(\beta-6)}}\left(x-\left(1-\frac{\beta}{6}\right) t\right)\right) \pm 1}, \\
& u_{373,374}(x, t)=-\frac{1}{2}-\frac{1}{4} \frac{\cosh \left(\frac{1}{2} \sqrt{\frac{\beta}{\alpha(\beta-6)}}\left(x-\left(1-\frac{\beta}{6}\right) t\right)\right) \pm 1}{\sinh \left(\frac{1}{2} \sqrt{\frac{\beta}{\alpha(\beta-6)}}\left(x-\left(1-\frac{\beta}{6}\right) t\right)\right)} \\
& -\frac{1}{4} \frac{\sinh \left(\frac{1}{2} \sqrt{\frac{\beta}{\alpha(\beta-6)}}\left(x-\left(1-\frac{\beta}{6}\right) t\right)\right)}{\cosh \left(\frac{1}{2} \sqrt{\frac{\beta}{\alpha(\beta-6)}}\left(x-\left(1-\frac{\beta}{6}\right) t\right)\right) \pm 1},
\end{aligned}
$$




$$
\begin{aligned}
& u_{375,376}(x, t)=-\frac{1}{2}+\frac{\sqrt{2}}{4} \frac{\cos \left(\frac{\sqrt{2}}{2} \sqrt{\frac{\beta}{\alpha(\beta-6)}}\left(x-\left(1-\frac{\beta}{6}\right) t\right)\right) \pm 1}{\sin \left(\frac{\sqrt{2}}{2} \sqrt{\frac{\beta}{\alpha(\beta-6)}}\left(x-\left(1-\frac{\beta}{6}\right) t\right)\right)} \\
& +\frac{\sqrt{2}}{4} \frac{\sin \left(\frac{\sqrt{2}}{2} \sqrt{\frac{\beta}{\alpha(\beta-6)}}\left(x-\left(1-\frac{\beta}{6}\right) t\right)\right)}{\cos \left(\frac{\sqrt{2}}{2} \sqrt{\frac{\beta}{\alpha(\beta-6)}}\left(x-\left(1-\frac{\beta}{6}\right) t\right)\right) \pm 1}, \\
& u_{377,378}(x, t)=-\frac{1}{2}+\frac{\sqrt{2}}{4} \frac{\cos \left(\frac{\sqrt{2}}{2} \sqrt{\frac{\beta}{\alpha(\beta-6)}}\left(x-\left(1-\frac{\beta}{6}\right) t\right)\right) \pm 1}{\sin \left(\frac{\sqrt{2}}{2} \sqrt{\frac{\beta}{\alpha(\beta-6)}}\left(x-\left(1-\frac{\beta}{6}\right) t\right)\right)} \\
& +\frac{\sqrt{2}}{4} \frac{\sin \left(\frac{\sqrt{2}}{2} \sqrt{\frac{\beta}{\alpha(\beta-6)}}\left(x-\left(1-\frac{\beta}{6}\right) t\right)\right)}{\cos \left(\frac{\sqrt{2}}{2} \sqrt{\frac{\beta}{\alpha(\beta-6)}}\left(x-\left(1-\frac{\beta}{6}\right) t\right)\right) \mp 1} \\
& u_{379,380}(x, t)=-\frac{1}{2}-\frac{\sqrt{2}}{4} \frac{\cos \left(\frac{\sqrt{2}}{2} \sqrt{\frac{\beta}{\alpha(\beta-6)}}\left(x-\left(1-\frac{\beta}{6}\right) t\right)\right) \pm 1}{\sin \left(\frac{\sqrt{2}}{2} \sqrt{\frac{\beta}{\alpha(\beta-6)}}\left(x-\left(1-\frac{\beta}{6}\right) t\right)\right)} \\
& -\frac{\sqrt{2}}{4} \frac{\sin \left(\frac{\sqrt{2}}{2} \sqrt{\frac{\beta}{\alpha(\beta-6)}}\left(x-\left(1-\frac{\beta}{6}\right) t\right)\right)}{\cos \left(\frac{\sqrt{2}}{2} \sqrt{\frac{\beta}{\alpha(\beta-6)}}\left(x-\left(1-\frac{\beta}{6}\right) t\right)\right) \pm 1} \\
& u_{381,382}(x, t)=-\frac{1}{2}-\frac{\sqrt{2}}{4} \frac{\cos \left(\frac{\sqrt{2}}{2} \sqrt{\frac{\beta}{\alpha(\beta-6)}}\left(x-\left(1-\frac{\beta}{6}\right) t\right)\right) \pm 1}{\sin \left(\frac{\sqrt{2}}{2} \sqrt{\frac{\beta}{\alpha(\beta-6)}}\left(x-\left(1-\frac{\beta}{6}\right) t\right)\right)} \\
& -\frac{\sqrt{2}}{4} \frac{\sin \left(\frac{\sqrt{2}}{2} \sqrt{\frac{\beta}{\alpha(\beta-6)}}\left(x-\left(1-\frac{\beta}{6}\right) t\right)\right)}{\cos \left(\frac{\sqrt{2}}{2} \sqrt{\frac{\beta}{\alpha(\beta-6)}}\left(x-\left(1-\frac{\beta}{6}\right) t\right)\right) \mp 1} \text {. }
\end{aligned}
$$

for $\frac{\beta}{\alpha(\beta-6)}<0$

$$
\begin{aligned}
& u_{383,384}(x, t)=-\frac{1}{2}+\frac{1}{2}\left(\frac{\sinh \left(\sqrt{\frac{-\beta}{\alpha(\beta-6)}}\left(x-\left(1-\frac{\beta}{6}\right) t\right)\right)}{\cosh \left(\sqrt{\frac{-\beta}{\alpha(\beta-6)}}\left(x-\left(1-\frac{\beta}{6}\right) t\right)\right) \pm 1}\right), \\
& u_{385,386}(x, t)=-\frac{1}{2}-\frac{1}{2}\left(\frac{\sinh \left(\sqrt{\frac{-\beta}{\alpha(\beta-6)}}\left(x-\left(1-\frac{\beta}{6}\right) t\right)\right)}{\cosh \left(\sqrt{\frac{-\beta}{\alpha(\beta-6)}}\left(x-\left(1-\frac{\beta}{6}\right) t\right)\right) \pm 1}\right), \\
& u_{387,388}(x, t)=-\frac{1}{2}+\frac{1}{2}\left(\frac{\cosh \left(\sqrt{\frac{-\beta}{\alpha(\beta-6)}}\left(x-\left(1-\frac{\beta}{6}\right) t\right)\right) \pm 1}{\sinh \left(\sqrt{\frac{-\beta}{\alpha(\beta-6)}}\left(x-\left(1-\frac{\beta}{6}\right) t\right)\right)}\right),
\end{aligned}
$$




$$
\begin{aligned}
& u_{389,390}(x, t)=-\frac{1}{2}-\frac{1}{2}\left(\frac{\cosh \left(\sqrt{\frac{-\beta}{\alpha(\beta-6)}}\left(x-\left(1-\frac{\beta}{6}\right) t\right)\right) \pm 1}{\sinh \left(\sqrt{\frac{-\beta}{\alpha(\beta-6)}}\left(x-\left(1-\frac{\beta}{6}\right) t\right)\right)}\right), \\
& u_{391,392}(x, t)=-\frac{1}{2}+\frac{i}{4} \frac{\cos \left(\frac{1}{2} \sqrt{\frac{-\beta}{\alpha(\beta-6)}}\left(x-\left(1-\frac{\beta}{6}\right) t\right)\right) \pm 1}{\sin \left(\frac{1}{2} \sqrt{\frac{-\beta}{\alpha(\beta-6)}}\left(x-\left(1-\frac{\beta}{6}\right) t\right)\right)} \\
& -\frac{i}{4} \frac{\sin \left(\frac{1}{2} \sqrt{\frac{-\beta}{\alpha(\beta-6)}}\left(x-\left(1-\frac{\beta}{6}\right) t\right)\right)}{\cos \left(\frac{1}{2} \sqrt{\frac{-\beta}{\alpha(\beta-6)}}\left(x-\left(1-\frac{\beta}{6}\right) t\right)\right) \pm 1}, \\
& u_{393,394}(x, t)=-\frac{1}{2}-\frac{i}{4} \frac{\cos \left(\frac{1}{2} \sqrt{\frac{-\beta}{\alpha(\beta-6)}}\left(x-\left(1-\frac{\beta}{6}\right) t\right)\right) \pm 1}{\sin \left(\frac{1}{2} \sqrt{\frac{-\beta}{\alpha(\beta-6)}}\left(x-\left(1-\frac{\beta}{6}\right) t\right)\right)} \\
& +\frac{i}{4} \frac{\sin \left(\frac{1}{2} \sqrt{\frac{-\beta}{\alpha(\beta-6)}}\left(x-\left(1-\frac{\beta}{6}\right) t\right)\right)}{\cos \left(\frac{1}{2} \sqrt{\frac{-\beta}{\alpha(\beta-6)}}\left(x-\left(1-\frac{\beta}{6}\right) t\right)\right) \pm 1}, \\
& u_{395,396}(x, t)=-\frac{1}{2}+\frac{\sqrt{2}}{4} \frac{\cosh \left(\frac{\sqrt{2}}{2} \sqrt{\frac{-\beta}{\alpha(\beta-6)}}\left(x-\left(1-\frac{\beta}{6}\right) t\right)\right) \pm 1}{\sinh \left(\frac{\sqrt{2}}{2} \sqrt{\frac{-\beta}{\alpha(\beta-6)}}\left(x-\left(1-\frac{\beta}{6}\right) t\right)\right)} \\
& -\frac{\sqrt{2}}{4} \frac{\sinh \left(\frac{\sqrt{2}}{2} \sqrt{\frac{-\beta}{\alpha(\beta-6)}}\left(x-\left(1-\frac{\beta}{6}\right) t\right)\right)}{\cosh \left(\frac{\sqrt{2}}{2} \sqrt{\frac{-\beta}{\alpha(\beta-6)}}\left(x-\left(1-\frac{\beta}{6}\right) t\right)\right) \mp 1}, \\
& u_{397,398}(x, t)=-\frac{1}{2}-\frac{\sqrt{2}}{4} \frac{\cosh \left(\frac{\sqrt{2}}{2} \sqrt{\frac{-\beta}{\alpha(\beta-6)}}\left(x-\left(1-\frac{\beta}{6}\right) t\right)\right) \pm 1}{\sinh \left(\frac{\sqrt{2}}{2} \sqrt{\frac{-\beta}{\alpha(\beta-6)}}\left(x-\left(1-\frac{\beta}{6}\right) t\right)\right)} \\
& +\frac{\sqrt{2}}{4} \frac{\sinh \left(\frac{\sqrt{2}}{2} \sqrt{\frac{-\beta}{\alpha(\beta-6)}}\left(x-\left(1-\frac{\beta}{6}\right) t\right)\right)}{\cosh \left(\frac{\sqrt{2}}{2} \sqrt{\frac{-\beta}{\alpha(\beta-6)}}\left(x-\left(1-\frac{\beta}{6}\right) t\right)\right) \mp 1} \text {. }
\end{aligned}
$$

Using (9), the solution of (10) when $p=\frac{k^{2}-2}{2}, q=\frac{k^{2}}{2}, r=\frac{1}{4}$, and the sets of solutions (3)-(10), we get

$u_{399,399, \ldots, 406}(x, t)=a_{0}+a_{1}\left(\frac{\mathrm{cn} \xi}{\sqrt{1-k^{2} \pm \operatorname{dn} \xi}}\right)+b_{1} \frac{\sqrt{1-k^{2}} \pm \operatorname{dn} \xi}{\operatorname{cn} \xi}$, where $a_{0}, a_{1}$ and $b_{1}$ are defined in the sets of solutions (3)-(10).

Note that, when $k \rightarrow 1$ we get constant solutions, when $k \rightarrow 0$ we obtain, $\left[u_{3,4}(x, t)\right.$ and $\left.u_{5,6}(x, t)\right]$. 


\section{Conclusion}

In this paper, the mapping method has been successfully implemented to find new traveling wave solutions for our new proposed equation namely, a combined Benjamin -Bona-Mahony (BBM) and modified Benjamin-Bona-Mahony (mBBM) equation. The results show that this method is a powerful Mathematical tool for obtaining exact solutions for our equation. It is also a promising method to solve other nonlinear partial differential equations.

\section{References}

[1] B. Ayhan and A. Bekir, "The $\left(\frac{G^{\prime}}{G}\right)$-expansion method for the nonlinear lattice equations," Communications in Nonlinear Science and Numerical Simulation, vol. 17, no. 9, pp. 3490-3498, 2012.

[2] V. O. Vakhnenko, E. J. Parkes, and A. J. Morrison, "A Backlund transformation and the inverse scattering transform method for the generalised Vakhnenko equation," Chaos, Solitons \& Fractals, vol. 17, no. 4, pp. 683-692, 2003.

[3] X. Q. Liu, S. Jiang, W. B. Fan, and W. M. Liu, "Soliton solutions in linear magnetic field and time-dependent laser field," Communications in Nonlinear Science and Numerical Simulation, vol. 9, pp. 361-365, 2004.

[4] R. Hirota, "Exact solution of Kortewege-de Vries equation for multiplecollisions of solitons," Physical Review Letters, vol. 27, pp. 1192-1194, 1971.

[5] G. Tanoglu, "Solitary wave solution of nonlinear multi-dimensional wave equation by bilinear transformationmethod," Communications in Nonlinear Science andNumerical Simulation, vol.12, no. 7, pp. 1195-1201, 2007.

[6] W. Malfliet, "Solitary wave solutions of nonlinear wave equations," American Journal of Physics, vol. 60, no. 7, pp. 650-654, 1992.

[7] A.-M. Wazwaz, "New solitons and kink solutions for the Gardner equation," Communications in Nonlinear Science and Numerical Simulation, vol. 12, no. 8, pp. 1395-1404, 2007.

[8] A.-M. Wazwaz, "A sine-cosine method for handling nonlinear wave equations," Mathematical and Computer Modelling, vol. 40, no. 5-6, pp. 499-508, 2004.

[9] A. Bekir, "New solitons and periodic wave solutions for some nonlinear physical models by using sine-cosine method," Physica Scripta, vol. 77, no. 4, article 501, 2008 .

[10] M. Wadati, H. Sanuki, and K. Konno, "Relationships among inversemethod, Backlund transformation and an infinite number of conservation laws," Progress of Theoretical Physics, vol. 53, pp. 419-436, 1975. 
[11] Z. Yan and H. Zhang, "Backlund transformation and exact solutions for (2+1)-dimensionalKolmogoroff-Petrovsky-Piscounov equation," Communications in Nonlinear Science \& Numerical Simulation, vol. 4, no. 2, pp. 146-151, 1999.

[12] M.Wang, "Exact solutions for a compound KdV-Burgers equation," Physics Letters A, vol. 213, no. 5-6, pp. 279-287, 1996.

[13] Z. Yan and H. Zhang, "New explicit solitary wave solutions and periodic wave solutions for Whitham-Broer-Kaup equation in shallow water," Physics Letters A, vol. 285, no. 5-6, pp. 355-362, 2001.

[14] V. B. Matveev and M. A. Salle, Darboux Transformations and Solitons, Springer Series in Nonlinear Dynamics, Springer, Berlin, Germany, 1991.

[15] S. K. Liu, Z. T. Fu, S.D.Liu, andQ. Zhao, "An expansionmethod by using the Jacobi elliptic functions and its applications to nonlinear wave equations," Acta Physica Sinica, vol. 50, no. 11, pp. 2068-2073, 2001.

[16] Y.-Z. Peng, "Exact solutions for some nonlinear partial differential equations," Physics Letters A, vol. 314, no. 5-6, pp. 401-408, 2003.

[17] A. Elgarayhi, "New periodic wave solutions for the shallow water equations and the generalized Klein-Gordon equation," Communications in Nonlinear Science and Numerical Simulation, vol. 13, no. 5, pp. 877-888, 2008.

[18] X. Zeng and X. Yong, "A new mapping method and its applications to nonlinear partial differential equations," Physics Letters A, vol. 372, no. 44, pp. 6602-6607, 2008.

[19] Maher, A., H. M. El-Hawary, and M. S. Al-Amry. "New Exact Solutions for New Model Nonlinear Partial Differential Equation." Journal of Applied Mathematics, (2013).

[20] Abbasbandy, S., and A. Shirzadi. "The first integral method for modified Benjamin-Bona-Mahony equation." Communications in Nonlinear Science and Numerical Simulation, pp. 1759-1764, (2010).

[21] Fetecau R, Levy D. "Approximate model equation for water waves." Comm. Math.Sci, pp. 159-170, 2005 . 